\title{
Cognitive Radio Dynamic Access Techniques
}

\author{
I. Budiarjo · M. K. Lakshmanan • H. Nikookar
}

Published online: 29 February 2008

(C) The Author(s) 2008

\begin{abstract}
The ever growing demand for wireless services has placed enormous burden on valuable resources such as spectral bandwidth. This has resulted in a major rethinking in resource allocation policies culminating in an explosion of research activity in the field of Cognitive Radio (CR) towards optimum resource usage. In this tutorial paper the physical layer design and transmission techniques for CR in the context of efficient spectrum utilization are discussed. Spectrum sensing as the key element of CR awareness is described. Orthogonal frequency division multiplexing (OFDM) as a spectrally efficient modulation scheme is discussed and the rationale for its use in the CR system is explained. Spectrum pooling for efficient use of spectrum is studied and the role of adaptive OFDM techniques in this method is highlighted. Wavelet basis function as a replacement of Fourier transform in OFDM is evaluated. MIMO system as an added value to the CR performance is described. Adaptive Waveform and beamforming as alternative techniques in CR are reviewed.
\end{abstract}

Keywords Adaptive bit loading - Beamforming - Cognitive radio · MIMO · OFDM . Spectrum pooling $\cdot$ TDCS $\cdot$ WDCS and Wavelet

This work was presented in part in 2nd CTIF Workshop, Aalborg University, Denmark, May 22-23, 2007.

I. Budiarjo ( $)$ · M. K. Lakshmanan · H. Nikookar

International Research Centre for Telecommunications and Radar, Department of Electrical Engineering, Mathematics and Computer Science, Delft University of Technology, Mekelweg 4, 2628CD, Delft,

The Netherlands

e-mail: i.budiarjo@irctr.tudelft.nl

M. K. Lakshmanan

e-mail: m.k.lakshmanan@tudelft.nl

H. Nikookar

e-mail: h.nikookar@irctr.tudelft.nl 


\section{Introduction}

\subsection{Background}

Advances in wireless technologies have spawned the development of a host of new and innovative wireless applications and services. With each passing day there is demand for more wireless services even when the popularity of existing applications is on the rise. As a result the clamor for valuable resources such as transmission spectrum has reached a shrill note. Paradoxically studies after studies conclude that large swathes of licensed frequency bands remain unused for most of the time and that spectrum congestion is more due to inadequate access techniques rather than non-availability [1,2]. This has sparked a debate in the Telecommunications circles on the need to revamp existing spectrum regulatory policies and introduce newer approaches. One such initiative is the idea of Cognitive Radio (CR), a new paradigm that promises opportunistic utilization of unused spectrum and efficient spectrum management. In [2] Haykin has defined CR as an intelligent wireless communication system that is cognizant of its environment, learns from it and adapts its internal states to statistical variations in the incoming RF stimuli by making changes in certain operating parameters in real time with objectives of highly reliable communications whenever and wherever needed, and efficient utilization of the radio spectrum. Modulation scheme, transmit power, channel coding, and carrier frequency are examples of parameters to be exploited in CR. The modulation scheme is chosen in such a way that the data is transmitted reliably using the least possible spectrum; in other words the modulation method must be spectrally efficient. Spectral efficiency is influenced by the noise and propagation condition. The latter varies with time due to environmental changes, hence the modulation scheme should be able to adapt to the channel propagation variation. It should also support multi node communication, as several nodes exist in the CR network. Spectral utilization is optimized by allowing rental (i.e., unlicensed) users to transmit and receive data over portions of spectra when primary (i.e., licensed) users are inactive. This is done in a way that the rental users (RUs) are invisible to the licensed users (LUs). In such a setting, the LUs are ordinary mobile terminals and their associated base stations. They thus do not possess much intelligence. The RUs, on the other hand, should possess the intelligence of sensing the spectrum and use whatever resources are available when they need them. At the same time, the RUs should give up the spectrum when a LU begins transmission.

\subsection{System Operation and Cognitive Cycle}

Figure 1 gives the blocks of a CR system. The three fundamental tasks of the system are:

1. Spectrum Estimation - Gauge the radio spectrum scenario and perform radio scene analysis.

2. Channel State Estimation and Predictive Modeling-Accurate and timely channel state information (CSI) at the transmitter is important for accurate power control, prediction of channel capacity, and scheduling.

3. System Reconfiguration-Based on the radio spectrum scenario and the channel state information, the system dynamically adapts transmission parameters such as power, spectrum, modulation scheme and data rate.

The spectrum estimator senses the spectrum and detects the presence of interference regions and spectrum holes and the channel estimator gauges the channel to derive the channel state information. Based on this information the transmitter adapts one or more of 


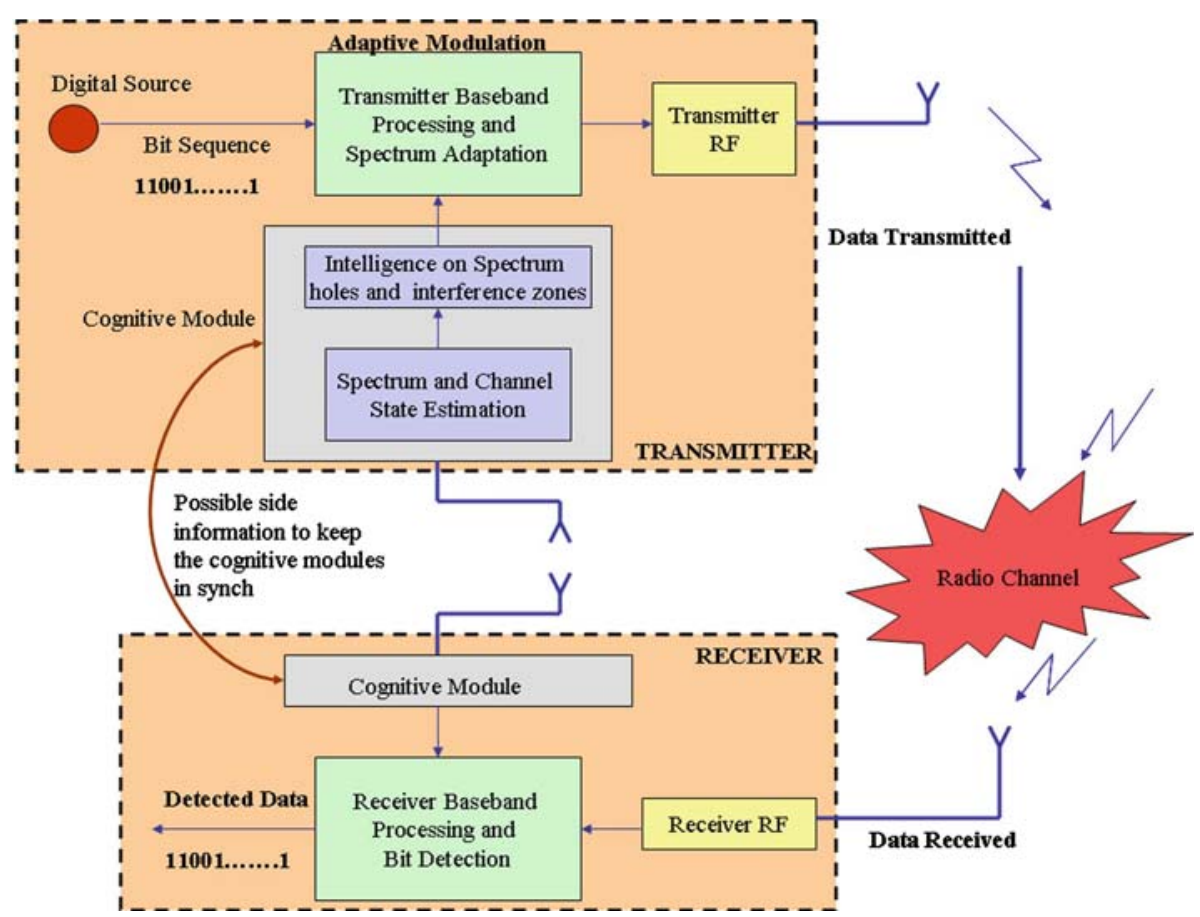

Fig. 1 System model of the Cognitive Radio transmitter: The major blocks include the spectrum and channel state estimator, adaptive pulse shaper (Transmission waveform shaping) and modulator. Receiver: The main components are the Signal base band processor and detector

the following: signal spectrum, modulation scheme, constellation size of code, data rate, and power. Consequently a pulse waveform that has little or no energy in the interference domains is constructed. The stream of data is modulated by the pulse waveform to obtain a transmission signal. The signal is then transmitted through the channel. At the receiver, the signal, corrupted by interference, is received and the data is detected using a suitable detector. Figure 2 illustrates the cognition cycle by which the system interacts with the environment and communicates.

\subsection{Techniques for Cognitive Radio}

Among several modulation schemes, Orthogonal Frequency Division Multiplexing (OFDM) is a suitable scheme that fulfils the requirements [3]. The cyclic prefix is inserted before the transmitted data that makes the scheme resistant to multipath fading, hence equalization is not required at the receiver. Multiple access can be applied by frequency division multiple access (FDMA) due to the carriers orthogonality, by time division multiple access (TDMA) where each node can access different OFDM symbols in a time frame, and multi carrier CDMA where node access is differentiated by orthogonal codes.

Recently a method called Spectrum Pooling is reported [4]. In spectrum pooling public access to spectrum is provided without sacrificing the transmission quality of the actual LUs. Multicarrier communication for CR has been suggested in [4]. The rationale is that any CR system needs to sense the spectrum, and this involves some sort of spectral analysis. Since FFT can be used for the spectral analysis [5] and at the same time it can act as the 


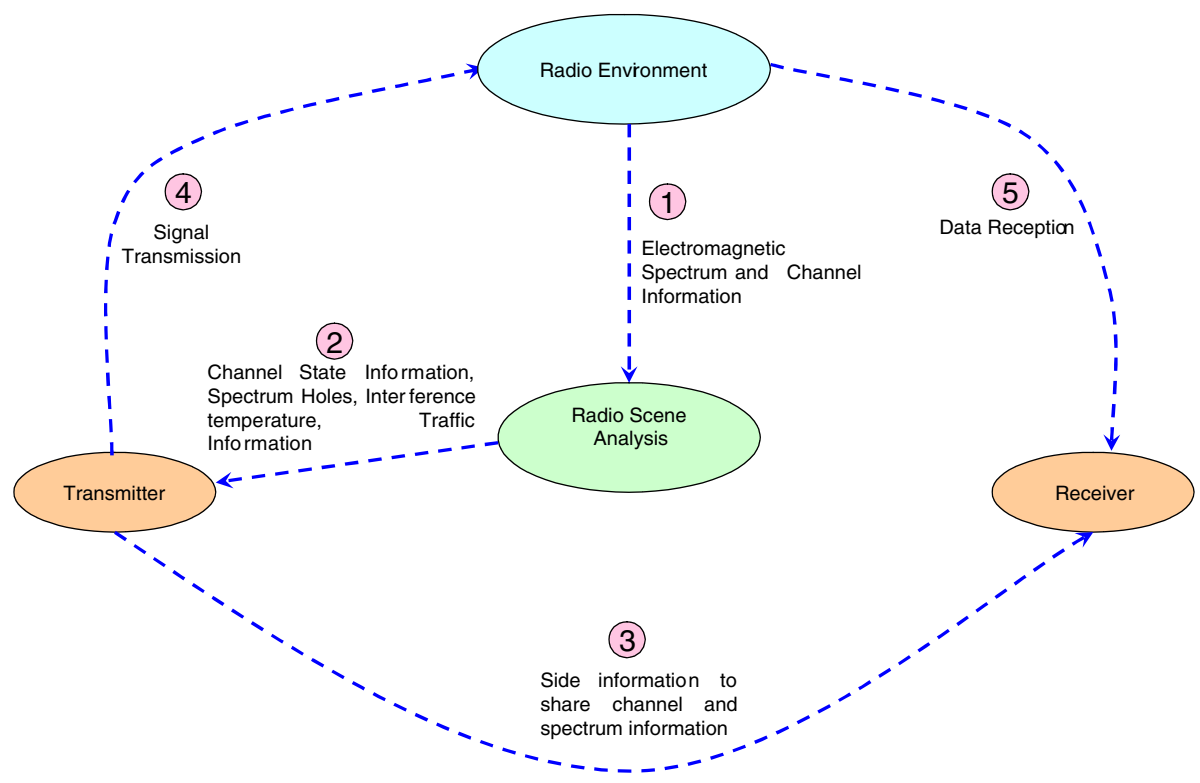

Fig. 2 Cognitive Radio signal transmission cycle

demodulator of an OFDM, it has been suggested as a proper candidate for multicarrier-based CR systems. A number of short-comings of OFDM in its application in CR have been noted in [4] and solutions to them have been proposed. The carriers located at the LUs band are deactivated, The sidelobes are reduced by deactivating more carriers adjacent to LUs band. Further sidelobes reduction is attained by applying windowing to the OFDM signal in time domain. If the number of available carriers for RU transmission is inadequate, due to lot of deactivations, then frequency hopping to another band is applied [6]. In [7] and [8] an interest of replacing Fourier transform by wavelet transform in multicarrier OFDM system is presented. The scheme gives better intercarrier interference (ICI) and inter symbol interference (ISI) reduction.

Single carrier CR method using adaptive waveform is proposed in [9] and [10]. In this method the transmitted signal is shaped in a way that its band does not contain the frequencies occupied by the LUs. The method takes the advantage of Fourier or wavelet transform.

\subsection{Theme and Organization of Paper}

In this paper spectrum sensing as the crucial aspect in CR system is discussed. Adaptive OFDM and its combination with spectrum pooling for improving the quality of service (QoS) and efficient use of the spectrum are reviewed. Wave shaping and beamforming as alternative methods of efficient utilization of the spectrum are addressed and their importance in CR is stressed. MIMO system as an added value to enhance the QoS of CR is described. The paper is structured as follows: Section 2 describes the spectrum sensing module as a spectrum awareness component of a CR system. The classification of various spectrum adaptation transmission techniques is discussed in Sect. 3. Due to the depth and importance of the topics, each of the spectrum adaptation techniques is discussed separately in individual sections. Multi carrier adaptive OFDM with Fourier and Wavelet basis functions and its combination 


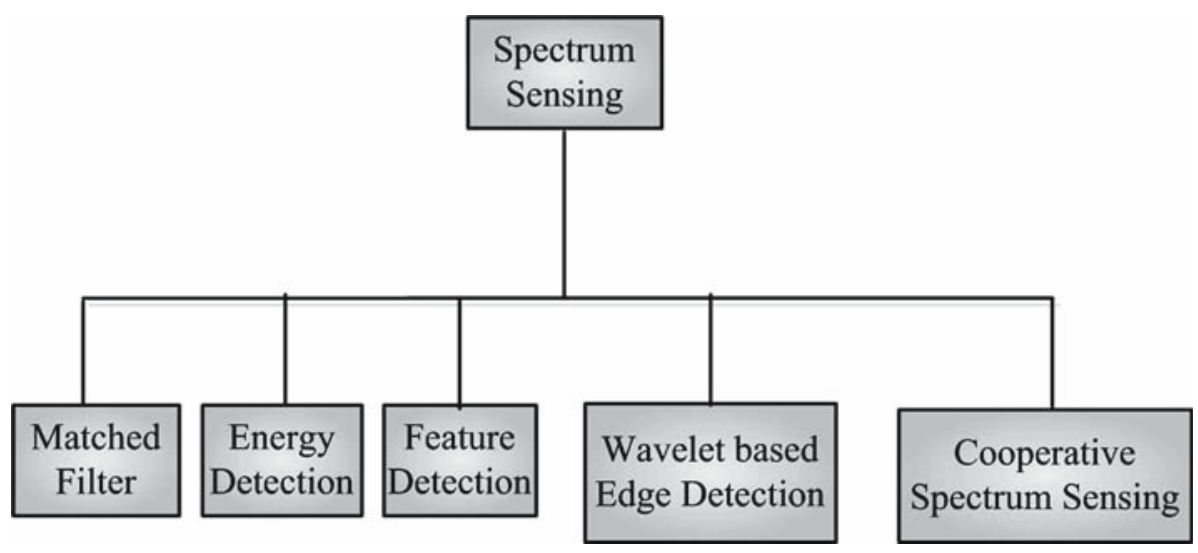

Fig. 3 CR spectrum sensing techniques

Fig. 4 Spectrum picture: $f_{c}$ is carrier frequency; $B$ is operation band [11]

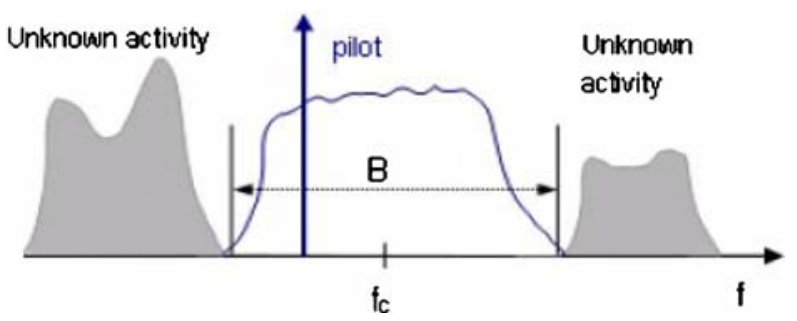

with spectrum pooling are analyzed in Sect. 4. Wave Shaping with single carrier modulation for CR is explained in Sect. 5. Section 6 shows the role of MIMO transmission in enhancing the CR performance. Beamforming as spatial interference avoidance technique in $\mathrm{CR}$ is discussed in Sect.7. A summary of the paper is given in Sect. 8.

\section{Spectrum Sensing}

The first and foremost task of any CR system is to gauge the wireless environments over wide frequency bands and identify the spectrum holes and occupied bands. This is done so that the CR system can opportunistically claim unused bands and operate invisibly without causing any distortion to other primary and LUs. The challenge of spectrum sensing module is in identification and detection of primary user signals amidst harsh and noisy environs. In this section we describe a few spectrum sensing techniques available in literature. Figure 3 gives a pictorial depiction of the various techniques available.

\subsection{Pilot Detection}

When prior information on the LU signal structure such as modulation scheme, pulse shape, packet format [5], band of operation $B$, center frequency $f_{c}$ [11] etc. are available, detection of pilots can be used to ascertain the presence or absence of a source (refer Fig. 4).

Such a scheme can be employed when communication systems operate with narrowband pilots channels. Examples of such systems would be TV signals (with narrowband audio/video carriers), CDMA systems (with pilot and paging channels), OFDM systems 


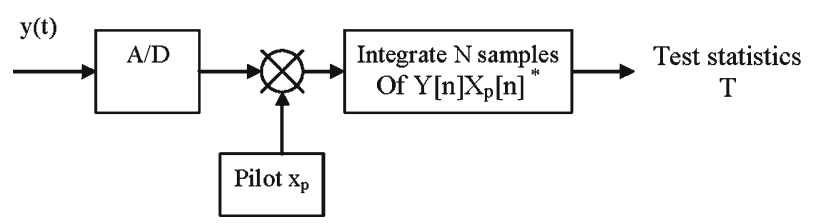

Fig. 5 Pilot detection using matched filter [11]

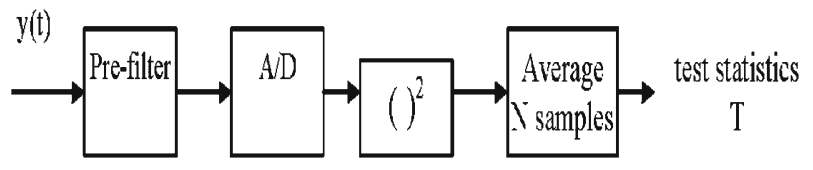

(a)

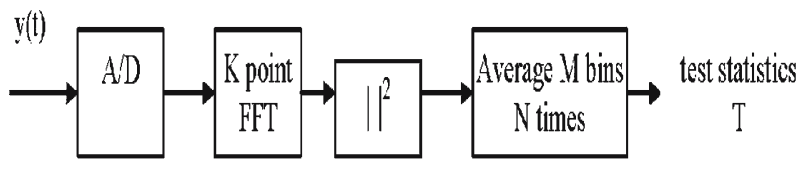

(b)

Fig. 6 Energy detector implementation with (a) Analog pre-filter and square law device, (b) Periodogram with FFT magnitude squared and averaged [5]

(with preambles) [5]. Figure 5 shows the implementation of a system to detect the pilot and thus the presence/absence of a primary source using a matched filter. Naturally, this method requires coherence with primary user signal and matched filter is a very good candidate for this technique because it maximizes the received signal to noise ratio (SNR) and is the optimal approach for signal detection and demodulation (Fig. 4). The drawback of such a method is that the $\mathrm{CR}$ will need a-priori knowledge on primary source characteristics and also a dedicated receiver for each $\mathrm{LU}$.

\subsection{Energy Detection}

Energy detection is a simpler sensing method than matched filtering as it is a non-coherent approach and hence does not need knowledge of pilot data.

Figure 6a shows the blocks of an energy detector. It consists of a low pass filter to weed out band noise and adjacent signal, an analog-to-digital (A/D) converter to digitize the signal and a square law device to calculate the energy. This implementation has been found to be ineffective for narrowband signals and sinewaves [11]. A more flexible and elegant approach is the periodogram where a Fourier transform of the received signal over a certain frequency band is taken and then averaged over an observation time to obtain the energy in the band (refer Fig. 6b). The frequency resolution can be improved by increasing the FFT point of the Fourier transform.

The disadvantage of the energy detection scheme is with setting a threshold to determine whether a frequency subband is occupied or not. Thresholds are difficult to define as they are susceptible to noise, fading and in-band interference. 


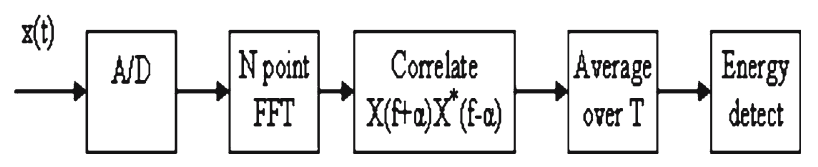

Fig. 7 Cyclostationary feature detector [5]. In the figure parameter $\alpha$ is the cycle frequency [5] and the power spectral density is derived when $\alpha=0$

\subsection{Cyclostationary Feature Detection}

The data generated by any digital source is random in nature, however, when the data is modulated with sinusoids (single carrier transmission), cyclic prefixes (multi-carrier transmission), hoping sequences (multiple access schemes), pulse trains, or code sequences (spread spectrum communication) a cyclostationarity results i.e. the modulated signals are characterized by statistics, mean and autocorrelation, that exhibit periodicity. The feature detection technique exploits this inherent cyclostationarity in modulated signals for detection of a wireless source. The implementation is depicted in Fig. 7. The cyclostationary feature detector can be designed as augmentation of the energy detector in Fig. 6. Number of signals, modulation types, symbol rates and the presence of interference are the features that can be detected with this technique [5].

\subsection{Wavelet Based Edge Detection}

Wavelets and wavelet transform are latest additions to the rich arsenal of communication system tool box. They possess excellent time-frequency localization properties that can serve as powerful mathematical tools to analyze local spectral structure to identify singularities and edges.

In [12] Z. Tian and G. B. Giannakis talk of a wavelet based wideband spectrum sensing approach for dynamic spectrum management. In their approach, the signal spectrum over a wide frequency band is decomposed into elementary building blocks of non-overlapping subbands that are well characterized by local irregularities in frequency. Then the entire wideband is modeled as a sequence of consecutive frequency subbands, where the power spectral characteristic is smooth within each subband but exhibit a discontinuous change between adjacent subbands. Information on the locations and intensities of spectrum holes and occupied bands is derived by considering the irregularities in power spectral density (PSD). The main attraction for wavelets in this application is in their ability to analyze singularities and irregular structures which can be used to characterize the local regularity and edges of signals. Hence, this name Edge detection.

Figure 8 gives an illustration of the method. It shows a snapshot of the PSD profile of the channel that typically a CR system will see. A typical wideband wireless network consists of heterogeneous devices using different wireless standards operating with different frequency ranges and centre frequencies. The goal of the CR is to spot a free band of say $B_{n}$ $\mathrm{Hz}$ bandwidth in the wideband frequency range $\left[f_{0}, f_{N}\right]$, where $N$ denotes the number of incumbent spectrum bands. Assuming that the CR received signal is $r(t)$ with $\operatorname{PSD} S_{r}(f)$, the edge detection problem is about finding $N,\left\{f_{n}\right\}$ and PSD of individual bands. Let $\phi(f)$ be a wavelet function and $\phi_{s}(f)$ a dilated version (by $\left.s\right)$ of $\phi(f)$, given by (1).

$$
\phi_{s}(f)=\frac{1}{s} \phi\left(\frac{f}{s}\right)
$$




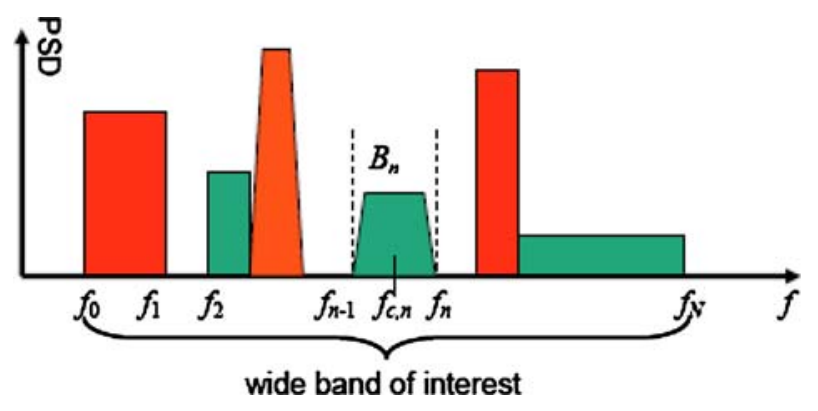

Fig. $8 N$ frequency bands with piecewise smooth PSD [12]

The continuous wavelet transform (CWT) of $S_{r}(f)$ is given as:

$$
C W T\left\{S_{r}(f)\right\}=S_{r} * \phi_{s}(f)
$$

An alternative way of finding the CWT of the PSD is to consider the auto-correlation $R_{r}(\tau)$ of the received signal $r(t)$ defined as:

$$
R_{r}(\tau)=E\{r(t) r(t+\tau)\}
$$

Using the notations FT for Fourier Transform and IFT for the Inverse Fourier Transform, the PSD and auto-correlation function is related as:

$$
S_{r}(f)=F T\left\{R_{r}(\tau)\right\}
$$

Defining, $\Phi_{s}(\tau)=\operatorname{IFT}\left\{\phi_{s}(f)\right\}$, it can be easily proved that the CWT of the PSD can be derived from the auto-correlation function as follows:

$$
C W T\left\{S_{r}(f)\right\}=F T\left\{R_{r}(\tau) . \Phi_{s}(s \tau)\right\}
$$

The signal irregularities and hence details of the spectrum holes and occupied bands can be characterized by spotting the local extrema of the first derivative and the zero-crossings of the second derivative. In particular, the local maxima of the wavelet modulus are sharp variation points, which tend to be more accurate than local minima points (corresponding to slow variation points) for spectrum sensing purposes.

A work on similar lines is carried out in [13] where a CR system architecture with a wideband dual-stage spectrum sensing technique, a coarse and a fine spectrum sensing, is suggested.

\subsection{Cooperative Spectrum Sensing and Beamforming}

The techniques described in the previous sections are local spectrum sensing schemes where individual CR nodes survey the spectrum to gather information. This approach would prove futile if the CR system was shadowed or the multipath fading is severe hampering accurate channel measurements [5]. Under these circumstances a more intuitive and suitable approach would be for a group of CR systems to co-operate gauge the channel together. Here each CR system broadcasts its snapshot of the spectrum to other units and a spectrum occupancy table is maintained to better regulate spectrum access. This way the local measurements of the landscape can be combined and channel conditions such as multi-path fading, shadowing and local interferences can be better handled. 
In [14] the advantage of spatial diversity is utilized to detect the strong LU that occupied a distinct frequency bands at spatial directions of arrival. This is due to the fact that spatially received signals occupy a limited number of directions or spatial clusters [14]. Beamforming as spatial sensing method and its transmission technique will be further studied in Sect. 7.

\section{Spectrum Adaptation}

Once enough knowledge on the spectrum holes and occupied zones are available, the next step in the cognition cycle is to adapt the transmission waveform in a way that the CR setup blends seamlessly amidst existing licensed operators. The goal of spectrum adaptation is to exploit knowledge of the electromagnetic environment to adapt its operations and access the unutilized spectrum. The key challenge in this process is in chiseling the wave shape to cover a wide bandwidth across varying dimensions of spectrum holes and occupied bands. The various spectrum adaptation schemes can be broadly classified into three techniques, namely, single carrier, multicarrier modulation and beamforming as spatial based spectrum adaptation technique. MIMO is an added value transmission technique applied either for single carrier or multi carrier spectrum adaptation to enhance the QoS of the CR system. Figure 9 depicts a classification of the various spectrum adaptation techniques available. In the next few sections we will delve on these spectrum adaptation schemes that facilitate opportunistic utilization of vacant spectral bands to communicate. Each of these techniques are unique and important in their own right warranting a dedicated section.

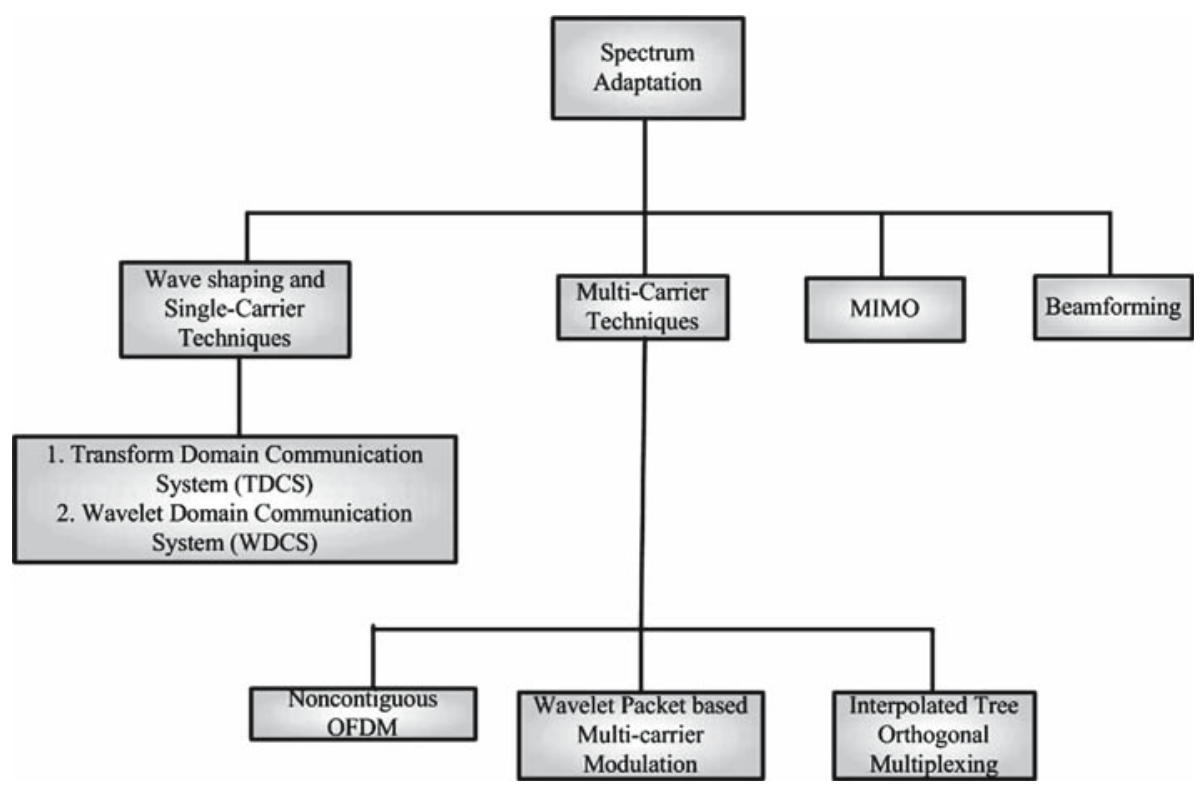

Fig. 9 CR spectrum adaptation techniques 


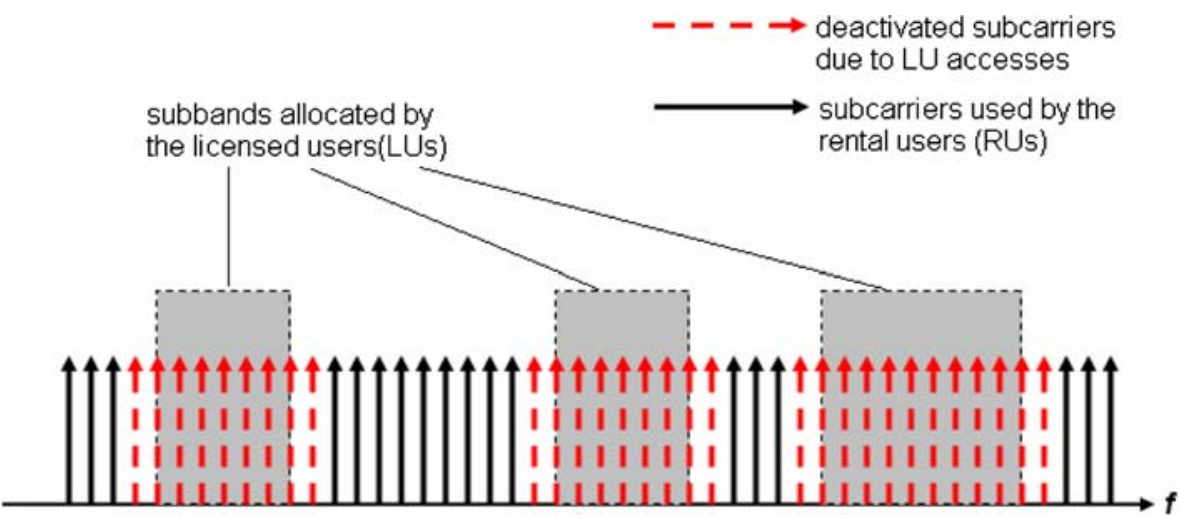

Fig. 10 Illustration of Spectrum pooling block diagram

\section{Cognitive Radio with Adaptive Multicarrier Modulation and Spectrum Pooling}

A strategy which is called spectrum pooling is proposed in [4] where the public access is enabled to these bands without giving significant interference to the actual license owners. Spectrum pooling enables public access to spectral ranges of licensed frequency bands which are seldom used by overlaying a secondary RU to an existing LU. The LUs are radio systems authenticated to operate under licensed spectral bands. The RUs are intelligent CR systems that actively scan the landscape and opportunistically utilize available and unused resources. The RU relinquishes control over the resources (here spectrum) as and when the LU starts using them. To identify and utilize unused bands, the frequency bands of various radio systems (including LUs and RUs) are combined to obtain a common spectral pool. Cohabitation of LU and RU systems is actualized by shaping the transmission waveform of the RU in a way that it utilizes the unoccupied time-frequency gaps of the LU.

The pioneering work on the subject was conducted by Jondral et al. [4] who devised a spectrum pooling scheme using multi-carrier modulation (MCM) where individual subcarriers adjacent to the occupied LU spectrum are deactivated for frequencies which are occupied by a LU. Figure 10 illustrates a typical scenario. The combination of adaptive MCM and spectrum pooling can serve as a robust method to achieve a good quality of communication and efficient use of the spectrum.

In the next subsections Fourier based (OFDM) and Wavelet based MCM with its application in Spectrum Pooling are explored.

\subsection{Fourier Based Multicarrier OFDM}

An obvious MCM candidate for CR systems is OFDM. With advantages of flexibility, ease of implementation and elegance in operation, OFDM is naturally suitable for CR system design. The short-coming of the OFDM solution is the large sidelobes of the frequency response of filters that characterize the channel associated with each of subcarrier. The sidelobes amplitude of the Fourier based OFDM can be observed from its PSD,

$$
P S D(f)=\frac{1}{N_{F F T}}\left|\sum_{m=0}^{N_{F F T-1}} \sqrt{p_{m}} A_{m} \int_{-(1+\alpha) \frac{T_{u}}{2}}^{(1+\alpha) \frac{T_{u}}{2}} g(t) e^{-j 2 \pi\left(f-f_{m}\right) t} d t\right|^{2}
$$


where $N_{F F T}$ is the FFT point, $T_{u}$ is the useful signal duration, $g(t)$ is the window function, $\alpha$ is the roll off factor of the window, $f_{m}$ is the frequency on carrier $m . p_{m}$ and $A_{m}$ are the allocated power and the symbol from QAM or PSK mapping on subcarrier $m$, respectively.

The large side lobes result in significant interference among the subcarriers that originate from different RUs and between LUs and RUs. Equation 6 shows that parameters like allocated power $\left(p_{m}\right)$, symbol $\left(A_{m}\right)$ amplitude and window $(g(t))$ can be set to resolve this problem. Other elements which can be varied to improve the performance of RUs are the channel coding parameters and carrier frequency. The utilization of the parameters to reduce the PSD in the LUs band and elements to improve the QoS of RUs will be further explored in the next following sub subsections.

\subsubsection{Adaptive Power Allocation}

The allocated power on the carriers adjacent to the LUs band can be set so the sidelobes becoming low, while no power is allocated to LUs band, hence the unallocated power can be distributed to other carriers.

The distribution can be applied equally or adaptively to the other carriers. Other options are to distribute the power only to data, or only pilots, or only to pilots/data which are not too close but also not too far from the LUs band with the intention not to largely increase the sidelobe to the LUs band but improve the signal to interference and noise ratio (SINR) of the pilots/data of RU on that position due to the possibility of the high sidelobes of LU to RU.

Adaptive power allocation is applied to the data or pilots based on the estimated channel gain. In [15] and [16] the power is allocated to each carrier with the objective of minimizing the overall BER. The allocated power to each carrier is derived from [15]:

$$
\begin{aligned}
p_{m}= & \frac{(C S-1) \sigma_{m}^{2}}{3 \Phi_{m}} W\left(\Phi_{m}^{2} \eta\right), \quad m=1, \ldots, N_{F F T} \\
& \text { subject to } \sum_{m=1}^{N_{F F T}} P_{m}=P_{T}
\end{aligned}
$$

where $C S$ is the constellation size of the symbol $A_{m}$ in (6), $\sigma_{m}^{2}$ is the noise variance on carrier $m, W(\cdot)$ is the Lambert function (i.e., inverse function of $\left.y(w)=w e^{w}\right), \Phi_{m}=\left|H_{m}\right|^{2}$ where $H_{m}$ is the channel gain on carrier $m, \eta$ is a constant and $P_{T}$ is the total allocated power per OFDM symbol.

In [17] optimal power allocation by steepest descent algorithm is introduced. In the beginning initial power to each carrier $\left(p_{m}(0)\right)$ and initial step size parameter $\mu(0)$ are set. The power is updated accordingly as [17]:

$$
p_{m}(i+1)=p_{m}(i)-\mu(i)\left(\frac{1}{N_{F F T}} \frac{\mathrm{d}}{\mathrm{d} p_{m}(i)} v\left(\frac{\Phi_{m} p_{m}(i)}{\sigma_{m}^{2}}\right)\right)+\lambda(i)
$$

where $i$ is the iteration number, $v(\cdot)$ is the BER function determined by the modulation mode used, and $\lambda(i)$ is determined by

$$
\lambda(i)=-\frac{1}{N_{F F T}^{2}} \sum_{m=1}^{N_{F F T}} \frac{\mathrm{d}}{\mathrm{d} p_{m}(i)} v\left(\frac{\Phi_{m} p_{m}(i)}{\sigma_{m}^{2}}\right)
$$

If all of the $p_{m}$ values are positive the new stepsize $\mu(i+1)$ is assumed as $\mu(i)$ and the power is further updated until the total power $P_{T}$ constraint in (7) is fulfilled. Otherwise the stepsize on specific carrier $m$ with negative $p_{m}$ is updated. The new $\mu_{m}$ becomes [17]: 


$$
\mu_{m}(i)=\frac{p_{m}(i)}{\frac{1}{N_{F F T}} \frac{\mathrm{d}}{\mathrm{d}_{p_{m}}(i)} v\left(\frac{\Phi_{m} p_{m}(i)}{\sigma_{m}^{2}}\right)+\lambda(i)}
$$

and the new stepsize $\mu(i)$ becomes the multiplication of a scaling factor $\rho(0<\rho<1)$ with the smallest $\mu_{m}$ [17]. The power to those carriers $\left(p_{m}\right)$ is updated again according to (8) until all $p_{m}$ s are nonnegative and $P_{T}$ constraint is achieved.

A suboptimal power allocation is also introduced in [17] by utilizing the upper bound approximation of the BER function $(v())$ as:

$$
v\left(\frac{\Phi_{m}}{\sigma_{m}^{2}} p_{m}\right) \leq \frac{(\sqrt{C S}-1)}{\sqrt{C S}} \log _{2}(\sqrt{C S}) e^{-\frac{3}{2(C S-1)} \frac{\Phi_{m}}{\sigma_{m}^{2}} p_{m}}
$$

By applying the BER upper bound in (11) combined with the total power $\left(P_{T}\right)$ constraint to the Lagrange optimization function, the power allocation becomes :

$$
p_{m}= \begin{cases}\frac{\lambda_{0} \sigma_{m}^{2}}{\Phi_{m}}-\frac{2(C S-1) \sigma_{m}^{2}}{3 \Phi_{m}} \ln \left(\frac{\sigma_{m}^{2}}{\Phi_{m}}\right), & \frac{\Phi_{m}}{\sigma_{m}^{2}} \geq e^{-\frac{3 \lambda_{0}}{2(C S-1)}} \\ 0, & \frac{\Phi_{m}}{\sigma_{m}^{2}} \leq e^{-\frac{3 \lambda_{0}}{2(M-1)}}\end{cases}
$$

where $\lambda_{0}$ is derived from:

$$
\lambda_{0}=\frac{N_{F F T} \bar{P}+\frac{2(C S-1)}{3} \sum_{m \in S}\left(\frac{\sigma_{m}^{2}}{\Phi_{m}}\right) \ln \left(\frac{\sigma_{m}^{2}}{\Phi_{m}}\right)}{\sum_{m \in S}\left(\frac{\sigma_{m}^{2}}{\Phi_{m}}\right)}
$$

$\bar{P}$ is the average power and $S$ is the set of carriers where its power is nonzero.

In [18] the optimal and suboptimal power allocations are combined with Coded Weak Sub Carrier Excision (CWSE) method, where the weak subcarriers are excluded from transmission. Power allocation with objective to maximize the bit rate subject to a finite total allocated power and minimizing the total power allocation subject to a finite target rate optimization problem are decribed in [19], while in [20] a look up table, to find rate-SNR operating point, and Lagrange multiplier bisection search method are applied to calculate the allocated power on each carrier.

\subsubsection{Spectrum Shaping by Time Domain Windowing}

In [4] extension of each OFDM block with long cyclic prefix and suffix samples and application of some windowing to reduce the side-lobes of the subcarrier channels are suggested. Obviously, this solution is at the cost of bandwidth loss because excessive time should be allocated to cyclic extensions that otherwise could be used for data transmission. Further in [4], interference reduction method is extended by deactivating subcarriers located adjacent to the licensed system (LS) which provides a kind of shield to the LUs. Due to the channel propagation influence, in OFDM bit errors are typically concentrated in a set of severely faded subcarriers. This fact indicates that there is possibility that the QOS cannot be maintained. Meanwhile, as mentioned before, the carriers resource is limited due to deactivation of RU carriers on the occupied LU band. Having adaptive OFDM in CR is thus a solution. The heavily faded and noisy subcarriers are excluded from transmission to improve the overall bit error rate (BER) of the system, and the loss of throughput is counteracted by applying higher order modulation modes to the subcarriers which have better SNR or BER. 
Commonly used window type is the raised cosine window, which is defined as [4]:

$$
g(t)= \begin{cases}\frac{1}{T_{u}} & 0 \leq|t| \leq \frac{T_{u}(1-\alpha)}{2} \\ \frac{1}{2 T_{u}}\left\{1+\cos \left[\frac{\pi}{\alpha T_{u}}\left(|t|-\frac{T_{u}(1-\alpha)}{2}\right)\right]\right\} & \frac{T_{u}(1-\alpha)}{2} \leq|t| \leq \frac{T_{u}(1+\alpha)}{2} \\ 0 & \text { Otherwise }\end{cases}
$$

where $\alpha$ is the roll off factor, and $T_{u}$ is the useful OFDM symbol interval (without guard interval). The shape of the window in the region of $\frac{T_{u}(1-\alpha)}{2} \leq|t| \leq \frac{T_{u}(1+\alpha)}{2}$ determines how rapid the OFDM spectrum goes down to zero. The PSD will hit zero on the frequencies in the interval of $1 / T_{u}$. Bartlett window is defined by Eq. 15, [21] while Better than Raised Cosine (BTRC) Window which gives the lowest sidelobes among the three windows is defined by Eq. 16 [22].

$$
\begin{gathered}
g(t)= \begin{cases}\frac{1}{T_{u}} & 0 \leq|t| \leq \frac{T_{u}(1-\alpha)}{2} \\
\frac{1}{2 T_{u}}-\frac{1}{T_{u}}\left[\frac{|t|}{\alpha T_{u}}-\frac{1}{2 \alpha}\right] & \frac{T_{u}(1-\alpha)}{2} \leq|t| \leq \frac{T_{u}(1+\alpha)}{2} \\
0 & \text { Otherwise }\end{cases} \\
g(t)= \begin{cases}\frac{1}{T_{u}}, & 0 \leq|t| \leq \frac{T_{u}(1-\alpha)}{2} \\
\frac{1}{T_{u}} e^{\left(-2 \ln 2 / \alpha T_{U}\right)\left[|t|-\left(T_{U}(1-\alpha) / 2\right)\right]} & \frac{T_{u}(1-\alpha)}{2} \leq|t| \leq \frac{T_{u}}{2} \\
\frac{1}{T_{u}}\left[1-e^{\left.\left(\frac{-2 \ln 2}{\alpha T_{u}}\right)\left[|t|-\frac{T_{u}(1-\alpha)}{2}\right]\right],}\right. & \frac{T_{u} \leq|t| \leq \frac{T_{u}(1+\alpha)}{2}}{2} \\
0 & \text { Otherwise }\end{cases}
\end{gathered}
$$

A window with lower sidelobes than BTRC which is called the Flipped-Inverse Hyperbolic Secant (farcsech) window is proposed in [23]. The window is designed according to (17),

$$
g(t)= \begin{cases}\frac{1}{T_{u}}, & 0 \leq|t| \leq \frac{T_{u}(1-\alpha)}{2} \\ \frac{1}{T_{u}}\left[1-\frac{1}{\alpha T_{u} \gamma} \operatorname{arcsec} h\left(\frac{1}{\alpha T_{u}}\left(\frac{T_{u}(1+\alpha)}{2}-|t|\right)\right)\right], & \frac{T_{u}(1-\alpha)}{2} \leq|t| \leq \frac{T_{u}}{2} \\ \frac{1}{T_{u}}\left[\frac{1}{\alpha T_{u} \gamma} \operatorname{arcsec} h\left(\frac{1}{\alpha T_{u}}\left(|t|-\frac{T_{u}(1-\alpha)}{2}\right)\right)\right], & \frac{T_{u}}{2} \leq|t| \leq \frac{T_{u}(1+\alpha)}{2} \\ 0 & \text { Otherwise }\end{cases}
$$

where $\gamma=2 \ln (\sqrt{3}+2) /\left(\alpha T_{u}\right)$. According to [24] the duration of OFDM signal should be $2 T_{u}$ in order to complete a total $2 N_{F F T}$ samples to preserve the orthogonality, and zeros are added on the region outside the window $g(t)$. The window designs by Eqs. 14-17 affect the transmitted signal, as the consequence an error floor will be formed. Figure 11a shows an example Bartlett window design according to (15) which affects the transmitted signal. 
Fig. 11 (a) Window design with influence to the transmitted signal (b) Window design without influence to the transmitted signal
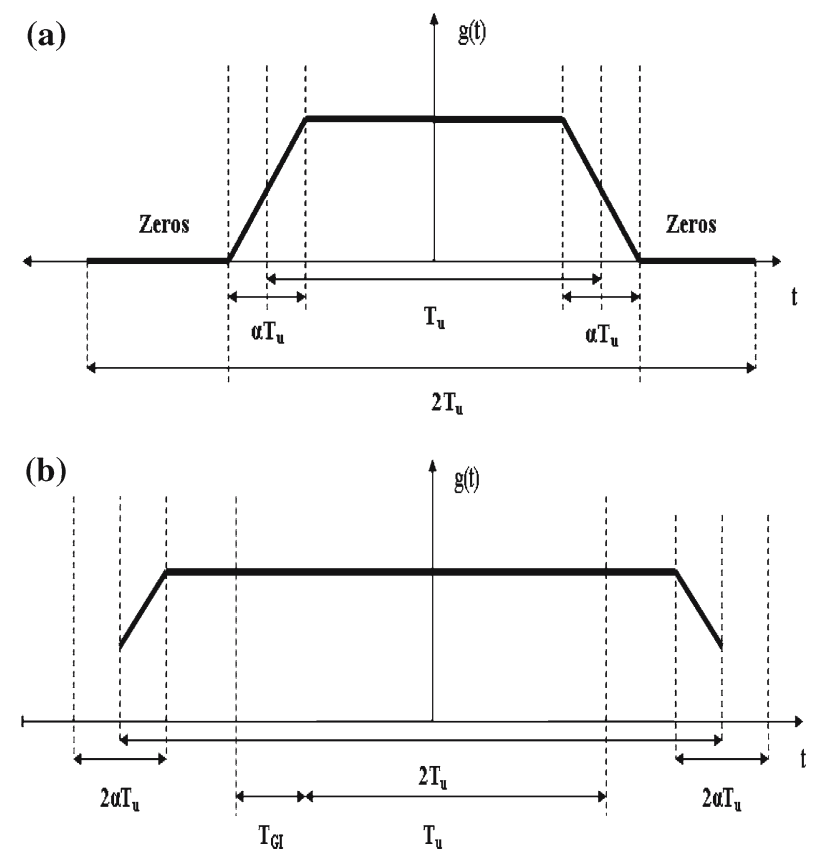

It is emphasized in [25] that the applied window must not influence the signal during its effective period to avoid error floor. In order to fulfil this requirement the window forms are expanded, i.e., new $T_{u}$ is becoming $2 T_{u}$, and $\alpha$ is restricted in the range of $0 \leq \alpha \leq$ $\left(1-T_{G I} / T_{u}\right) / 2$, where $T_{G I}$ is the guard interval duration. The orthogonality is preserved since the PSD hits zeros in the interval of $1 /\left(2 T_{u}\right)$ at the cost of longer duration of OFDM signal $\left(2 T_{u}(\alpha+1)\right)$. As a solution the window is truncated to fit $2 T_{u}$ OFDM duration, as depicted in Fig. 11b. The orthogonality is preserved by applying the rectangular receiver filter with duration $T_{u}$, implemented by DFT [25]. In this way there is more freedom in choosing window in the region of $T_{u}(1-\alpha) \leq|t| \leq T_{u}$ as long as its PSD on the symbol boundaries is low, e.g Gaussian window or half sine window [26].

It should be emphasized that applying only windowing is not enough to reduce interference to LU. More powerful technique to suppress OFDM sidelobes is carriers deactivation. Deactivation of carriers adjacent to the licensed band provides flexible guard bands which will make the PSD sidelobes of RUs OFDM signal on the licensed band lower. Applying only one of the methods may not be sufficient, therefore combining both methods (windowing and adaptive deactivation of carriers adjacent to licensed bands) is suggested to make the PSD sidelobes even lower in the region of LUs band. The loss of throughput due to carriers deactivation and long OFDM symbol duration can be compensated by applying adaptive bits allocation. The next sub subsection will explore the application of adaptive bit allocation in CR system including its available algorithms.

Recently in [27] overlapped OFDM symbol transmission with long symbol duration is proposed to counteract the throughput loss. The scheme is described in Fig. 12.

The delay between one OFDM symbol to another should be designed in such a way that no intersymbol interference occurs, which means the next OFDM symbol should start after the end of the previous useful data part of an OFDM symbol. If zeros are inserted at the 


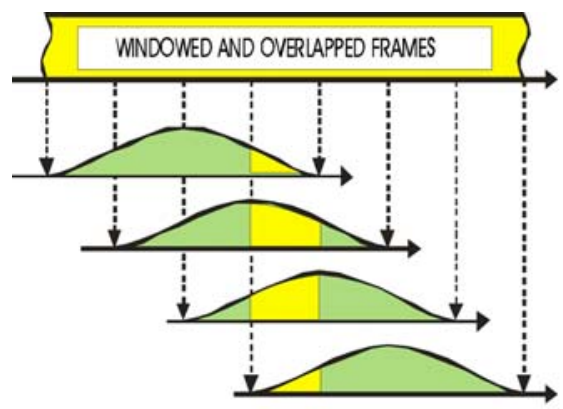

Overlapped Frame Time Intervals at Modulator

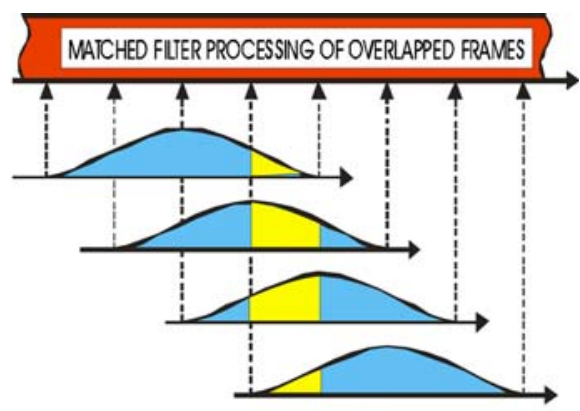

Overlapped Frame Time Intervals at Demodulator

Fig. 12 Overlapped OFDM symbol with zero ISI

prefix of suffix (outside the $T_{G I}$ and $T_{U}$ area as depicted in Fig. 11b), the useful data of the next OFDM symbol should start after the last useful data of the previous OFDM symbol.

\subsubsection{Adaptive Bit Allocation (Bit Loading)}

Referring to (6) the sidelobes to LUs band can be lowered by applying the low constellation size (e.g BPSK) to the symbols adjacent to LUs band. Another advantage of the low constellation size is its resistance to noise and interference compared to high constellation size. Hence, although the sidelobes of LU to RUs band exists, the BER of the RUs carriers adjacent to LUs band can be better preserved.

Adaptive bit loading is one of adaptive OFDM methods which allocates bits to subcarriers efficiently by taking estimated channel fading and noise variance information, and accordingly selecting the subcarriers modulation modes By utilizing the unused power due to carriers deactivation on the subband occupied by the LUs and allocating them to some of the other carriers intelligently, according to the adaptive bit loading algorithms, reliable communications without sacrificing the legacy system can be achieved. Transmitting more bits on a carrier requires more power. This power can be derived from the unallocated ones.

As a consequence of applying adaptive bit loading, the receiver needs information about the number of bits allocated on each carrier in order to decode the signal properly. This information can be derived blindly by examining the received signal $[28,29]$ or from dedicated signaling symbols. An example of the signaling symbol is depicted in Fig. 13, where the allowable constellation size is restricted to the set of $\{0,2,4,16$ and 64\}, [30]. The inner rectangle which is the decision area for 0 modulation mode (no symbol is transmitted) can be made smaller up to a distance of about $3 d / 4$ from the other reference symbols, where $d$ is the distance between outer points and the no symbol point shown in Fig. 13. This is due to the fact that a decision error (e.g no symbol transmitted but BPSK or other modulation mode is decided) is only caused by the noise (no contribution from channel fading).

Fischer-Hueber algorithm allocates the bits with the intention to minimize the BER on each carrier. It tries to guarantee equal SNR on every carrier. The bits are assigned as [31]:

$$
R_{m}=\frac{1}{K}\left[R_{T}+\log _{2}\left(\prod_{n=0}^{K-1} \frac{\sigma_{n}^{2}}{\Phi_{n}}\right)-K \log _{2}\left(\frac{\sigma_{m}^{2}}{\Phi_{m}}\right)\right]
$$




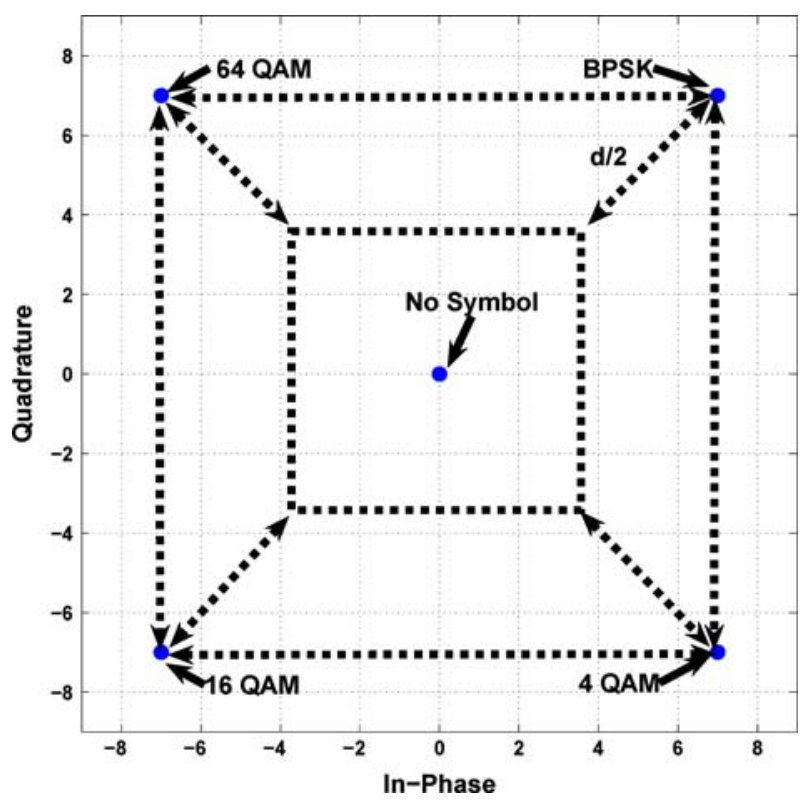

Fig. 13 An example of signaling symbol points for adaptive bit loading with allowable $M=\{0,2,4,16$, and 64\} [30]

where $R_{m}$ is the number of allocated bits on carrier $m, K$ is the number of active carriers, and $R_{T}$ the total target bits per OFDM symbol. In the beginning value of $K$ is equal to $N_{F F T}$, after applying (18) on each carrier the nonpositive values of $R_{m} \mathrm{~s}$ are replaced by zeros, and the number of active carriers $K$ is subtracted by the number of carriers with nonpositive $R_{m}$ values. The loop of calculating (18) continues until all $R_{m} \mathrm{~s}$ are nonnegative. Each of the $R_{m}$ is quantized into an integer number $R_{Q m}$. If the sum of the $R_{Q m}$ is not equal to the target rate, addition or subtraction of bit is applied. It starts from the carrier with the lowest deviation of $R_{Q m}$ to its $R_{m}$ (for the addition) or the carrier with the highest deviation (for the subtraction). The addition or subtraction is iteratively applied bit per bit until the target rate is fulfilled.

Another adaptive bit allocation algorithm is the Chow algorithm introduced in [32]. The goal is to maximize the allowable noise margin while the minimum desired BER is still achieved. The bits are assigned as follows [32]:

$$
R_{m}=\log _{2}\left[1+\frac{p_{m} \Phi_{m}}{\sigma_{m}^{2} \cdot\left(\Gamma+\gamma_{\text {margin }}\right)}\right],
$$

where $\gamma_{\text {margin }}$ is the additional amount of noise (in $\mathrm{dB}$ ) that the system can tolerate, while still achieving the minimum desired BER requirement.

The SNR gap $\Gamma$ is a constant which estimates the difference between channel capacity and the actual capacity usage by the transmission scheme, which is defined as:

$$
\Gamma=\frac{1}{3}\left[Q^{-1}\left(\frac{S E R}{4}\right)\right]^{2} \text {. }
$$

SER is the expected symbol error rate, and $Q(x)$ is the function $Q(x)=\frac{1}{\sqrt{2 \pi}} \int_{x}^{\infty} e^{\frac{-t^{2}}{2}} \mathrm{~d} t$.

Besides allocating the bits carrierwise, the bits can be allocated groupwise according to the average channel gain of the carriers within a certain group. This method reduces the 
Fig. 14 SNR grid for Simple Blockwise Loading Algorithm (SBLA) [34]

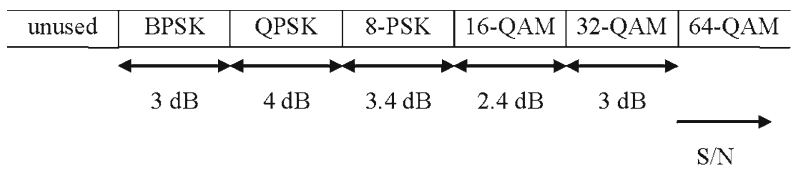

signaling symbols required to determine the modulation mode on each carrier, as the required modulation information of a group of $N_{c g}$ carriers is the same in a signaling symbol.

A Simple Blockwise Loading Algorithm (SBLA) is proposed in [33]. The algorithm divides the subcarriers into $N_{B}$ blocks of $N_{c b}$ adjacent subcarriers. The same modulation level is assigned to subcarriers within one block. The SNRs of neighboring sub carriers are highly correlated; hence no significant performance degradations are expected by introducing blocks of subcarriers, which are smaller than the coherence bandwidth of the channel. The choice of the modulation level for the specific block of subcarriers is based on the mean SNR of the block. Initially, the SNR grid is predetermined as e.g depicted in Fig. 14 [34]. The grid contains a list of differences of the SNR thresholds, required to switch between the modulations levels of the supported PSK/QAM modulation alphabets for a specific block. The middle point (center of 8-PSK) is placed on the overall SNR average of OFDM frame.

In [35] the Fischer-Hueber algorithm is modified to determine the allocated bits on a block of subcarriers. The bits are distributed as:

$$
R_{i}=\frac{R_{T}}{N_{B}^{\prime}}+\frac{1}{N_{B}^{\prime}} \log _{2}\left(\frac{\left(\left|H_{i}\right|^{2}\right)^{N_{B}^{\prime}}}{\prod_{k \in \psi}\left|H_{k}\right|^{2}}\right)
$$

where $R_{i}$ is the allocated bits to carriers block $i, N_{B}^{\prime}$ is the number of active blocks, $H_{i}$ is the average channel gain in block $i$, and $\psi$ is the set of active carriers block.

Like in the subcarrierwise Fischer-Hueber algorithm, after several iterations, the value of all bit allocations are non negative, but if the target rate is not yet achieved, the addition or substraction of bit to each carrier based on the deviation between the quantized bit allocation and unquantized bit allocation for Chow, SBLA and groupwise Fischer algorithm, is applied until the target rate is satisfied.

In [36] these bit loading algorithms are evaluated and compared by utilizing the $2 \times 1-\mathrm{D}$ Wiener filter as the channel estimation technique [37]. In [38] the performance of combination of adaptive bit loading algorithms and windows with the design according to Fig. 11b are evaluated. Simulation results in [37] and [38] show that carrierwise bit loading outperforms the groupwise bit loading, since the method maps the bit allocation to each carrier more accurately. If throughput is a critical issue, the groupwise bit loading is a better candidate than carrierwise bit loading. This is because in adaptive OFDM signaling symbols required by the receiver to recognize the modulation mode of each carrier, reduces the throughput, and groupwise bit loading requires less signaling symbols than the carrierwise.

\subsubsection{Adaptive Coding and Frequency Hopping}

An element which does not influence the level of interference to LUs band but is needed to boost or improve the QoS of CR system is the channel coding. As it can be noticed, none of the parameters shown in (6) refers to channel coding element. Most of references such as [39-42] discuss the adaptation of channel coding by varying the rate according to the estimated channel gain. The coding rate level is decreased as the channel fading power is increased [39]. 


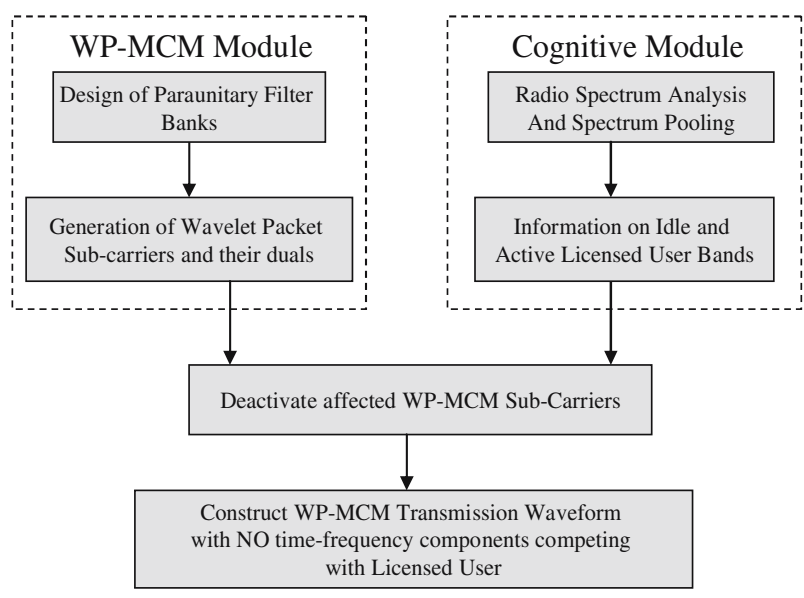

Fig. 15 Fundamental Blocks of WP-MCM based Cognitive Radio system. The level and the number of bases generated is given as $M=2^{J}$. A level 3 tree structure generates up to 8 wavelet packet carriers and duals each

In the situation where due to LUs access and bad channel condition, too many carriers are deactivated, thus the frequency hopping is suggested. The scanning module will give information to the front end to switch transmission to a certain carrier frequency where available spectrum in that region is adequate to achieve the target QoS. The performance of OFDM based CR using Wiener filter channel estimation with frequent frequency hopping at GSM $900 \mathrm{MHz}$ channel model is evaluated in [43], where the channel models are based on ETSI GSM specification for rural area and urban area power delay profiles.

\subsection{Wavelet Based Multicarrier OFDM}

In traditional implementations of Multicarrier OFDM (MC-OFDM), the generation and modulation of the sub-channels are accomplished digitally using Fourier bases. In this subsection another modulation technique is introduced. The conventional Fourier-based complex exponential carriers of OFDM are replaced with orthonormal wavelet packet (WP) bases for use in cognitive radio environments. The WP bases are derived from perfect reconstruction two-band FIR filter bank solutions [7,8,44,45].

Cohabitation of the WP-MCM based CR systems with existing LUs is actualized by shaping its transmission waveform and by adaptively activating or deactivating sub-carriers in a way that it utilizes the unoccupied time-frequency gaps of the LU. The idea is to dynamically sculpt the transmission signal so that it has no or very little time-frequency components competing with the LU. This way the CR can seamlessly blend with the LU operation. The steps to generate the WP-MCM transmission signal are elucidated in Fig. 15.

\subsubsection{Generation of Wavelet Packet Bases and Their Duals Through Two Channel Filter Bank Analysis}

The WP-MCM transmission is realized by replacing orthonormal complex exponential basis functions, as used in OFDM systems, with orthonormal wavelet packet basis functions. The wavelet packet bases and their dual bases are derived from perfect reconstruction two-band FIR filter bank solutions from multistage tree-structured paraunitary filter banks derived by cascading 2-channel filter banks. 


\subsubsection{Generation of Wavelet Packet Sub-Carrier Bases}

The wavelet packet sub-carriers (to be used at the transmitter end) are generated through a multichannel filterbank consisting of cascaded two-channel filterbanks applying the synthesis filters $\left(H^{\prime}\right.$ and $\left.G^{\prime}\right)$. The process known as the synthesis procedure consists of binary interpolation (up-sampling) by 2 , filtering and recombination at each level.

To demonstrate the process of generation of wavelet packet bases, here we consider a cascaded level-3 tree structure as shown in Fig. 16a. Such an arrangement can give rise to eight wavelet packet bases. This procedure is simplified further by applying the identities shown in Fig. 16b. These expressions called the noble identities are popularly applied in the implementation of multi-rate systems. And in Fig. 16c the equivalent eight-channel system after the application of the noble identities is shown. In general, to generate $M$ bases or sub-carrier waveforms, a level- $J$ tree has to be constructed.

\subsubsection{Generation of Wavelet Packet Dual Bases}

The wavelet packet duals (to be used at the receiver end) are obtained from multichannel filter bank analysis too, though the processes are reversed. The duals are obtained from the analysis filters $(H$ and $G)$ through the analysis procedure which consists of filtering, decimation (downsampling) by 2 and decomposition at each stage. Figure 17 illustrates the generation of eight wavelet packet duals from a level-three tree cascaded filter bank.

\subsubsection{Wavelet Design Considerations for WP-MCM Application}

Choosing the right wavelet is a delicate and at times even an overwhelming issue. In theory any time and frequency limited function can be utilized. However in practice, the wavelet bases cannot be arbitrarily chosen and instead have to satisfy a number of requirements.

With regard to the applicability to WP-MCM systems, the desirable properties may be listed as follows:

- The bases must be time and band limited

- The bases and their duals must be orthogonal to one another to enable perfect reconstruction.

- The bases must be orthogonal to one another in order to have unique demodulation.

Considering these requirements, among several available wavelet such as: Coifflets, Daubechies, Haar, Symlets, the most suitable wavelet is the family of maximally frequency filter banks derived using a modified Remez exchange algorithm. It provides degree of freedom to shape its sidelobes by varying its regularity order, transition bandwidth and the filter length. The longer the filter length the more degree of freedom available in designing the frequency selectivity and also the regularity order of the signal. The smaller the transition bandwidth the more frequency selective the filter will be. Regularity corresponds to the sidelobes of the signal. The higher the regularity order, the smoother or the lower the sidelobes of the signal. More on this subject can be found in [46]. In [47] WP-MCM with Remez exchange algorithm as CR system is evaluated and compared with Fourier based MC-OFDM CR system in the presence of LU signal in Additive White Gaussian Noise (AWGN) channel. The results show within a certain Remez parameters value, WP-MCM outperforms the Fourier based MC-OFDM.

\subsection{Interpolated Tree Orthogonal Multiplexing}

In the WP-MCM technique the filter banks perform the dual role of shaping the spectrum as well as interpolating in time series. A more intuitive method is to separate the two 
(a)

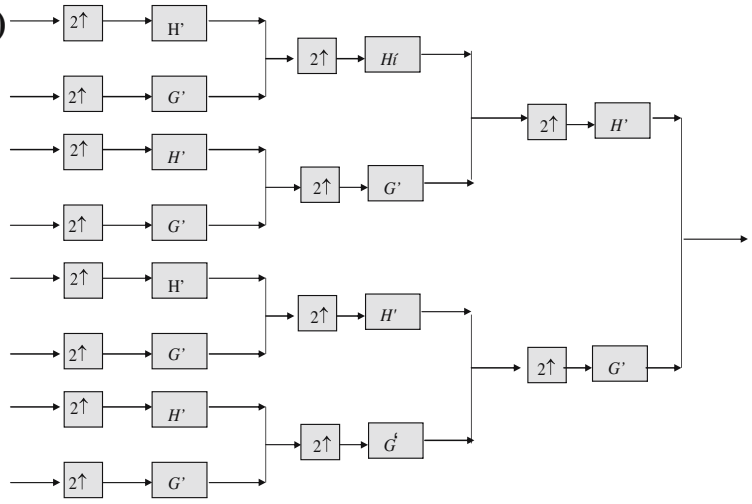

(b)
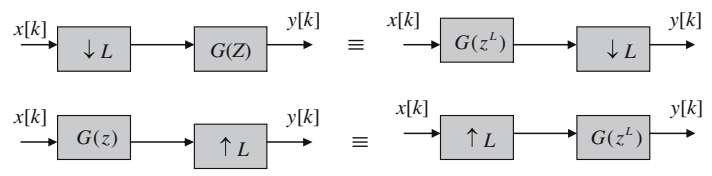

(c)

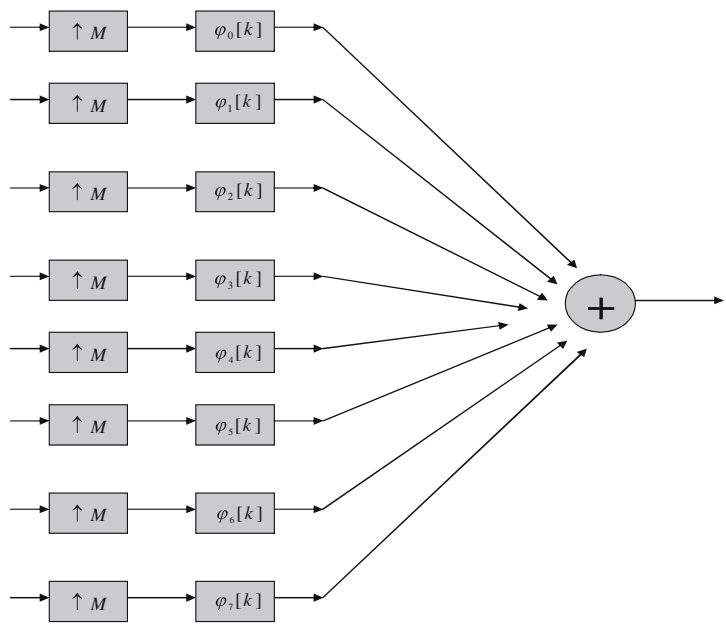

Fig. 16 Generation of wavelets. (a) A level-3 tree gives eight wavelet packet bases. The up arrows represent interpolation by $2 . H^{\prime}$ and $G^{\prime}$ denote the frequency responses of the low and high pass reconstruction filters, respectively; (b) Noble identities; (c) Equivalent eight-channel system after applying the noble identities, where $\varphi^{\prime}$ is equivalent reconstruction filter as results of the convolution combination $H^{\prime}$ and $G^{\prime}$ according to the tree lines. $M=8$ for the example considered

processes to have a greater control over the characteristics of the carriers. This method called the Interpolated Tree Orthogonal Multiplexing (ITOM) was introduced in [27] by constructing a binary wavelet packet tree and performing the spectral shaping external to the tree structure. The procedure is depicted in Fig. 18 from which we can notice that up-sampled shaping filters precede the input ports of the wavelet packet tree structure. Notching over the desired spectral interval is achieved by vacating one or more of the input branches. 

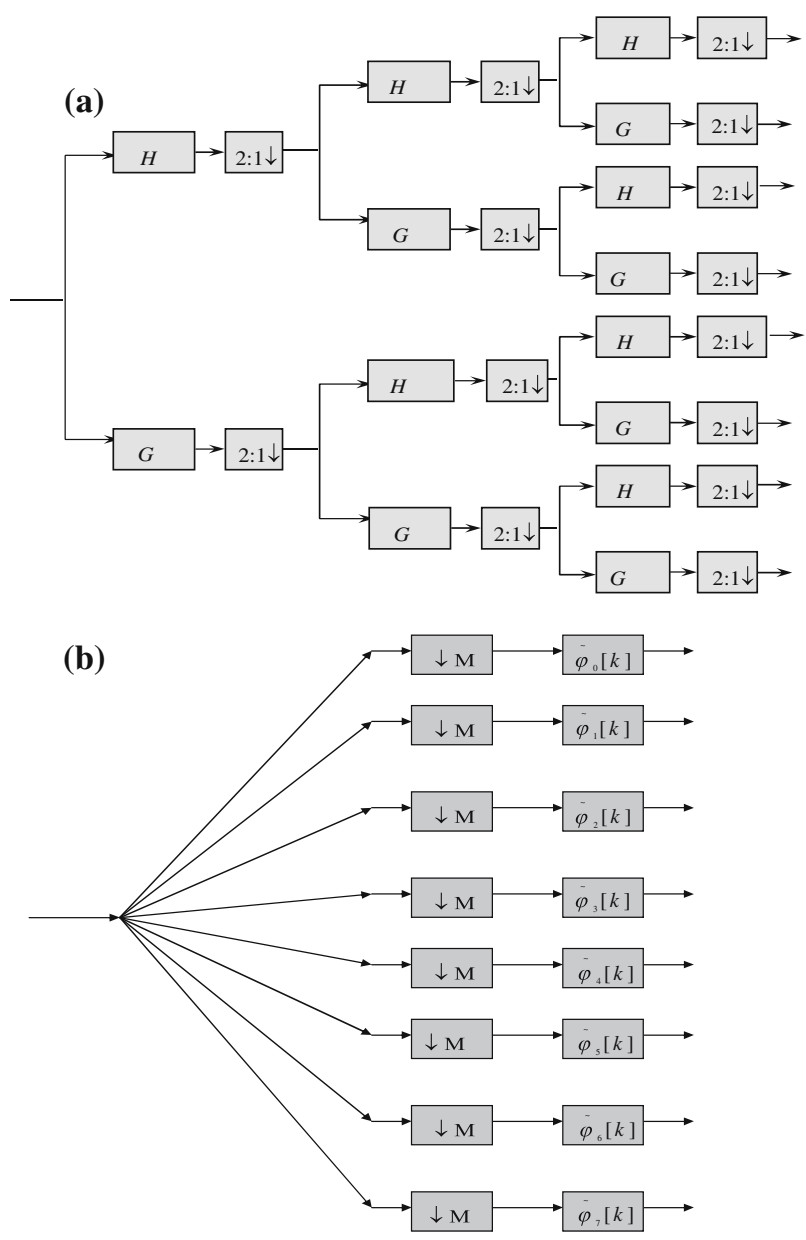

Fig. 17 Generation of wavelet Duals. (a) A level-three tree gives eight wavelet packet dual bases. The down arrows represent decimation by $2 . H$ and $G$ denote the frequency responses of the low and high pass decomposition filters, respectively; (b) Equivalent eight-channel system after applying the noble identities, where $\varphi$ is equivalent decomposition filter as results of the convolution combination $H$ and $G$ according to the tree lines $M=8$ for the example considered

Figure 19 shows an example of the ITOM mechanism. We may note from Fig. 19a and b that the enabled and disabled carries neatly fit into the spectral gaps of one-another illustration the superiority of the ITOM procedure towards spectrum shaping.

\section{Single Carrier Cognitive Radio with Adaptive Waveform}

Another method of utilizing the spectrum efficiently is shaping of the waveform of the transmitted signal dynamically over a spectral region, in order not to interfere with the occupied spectrum. Transform Domain Communication System (TDCS) is introduced in [9] as a candidate technique for $\mathrm{CR}$. In principle the transmitted signal is shaped in such a way that its 


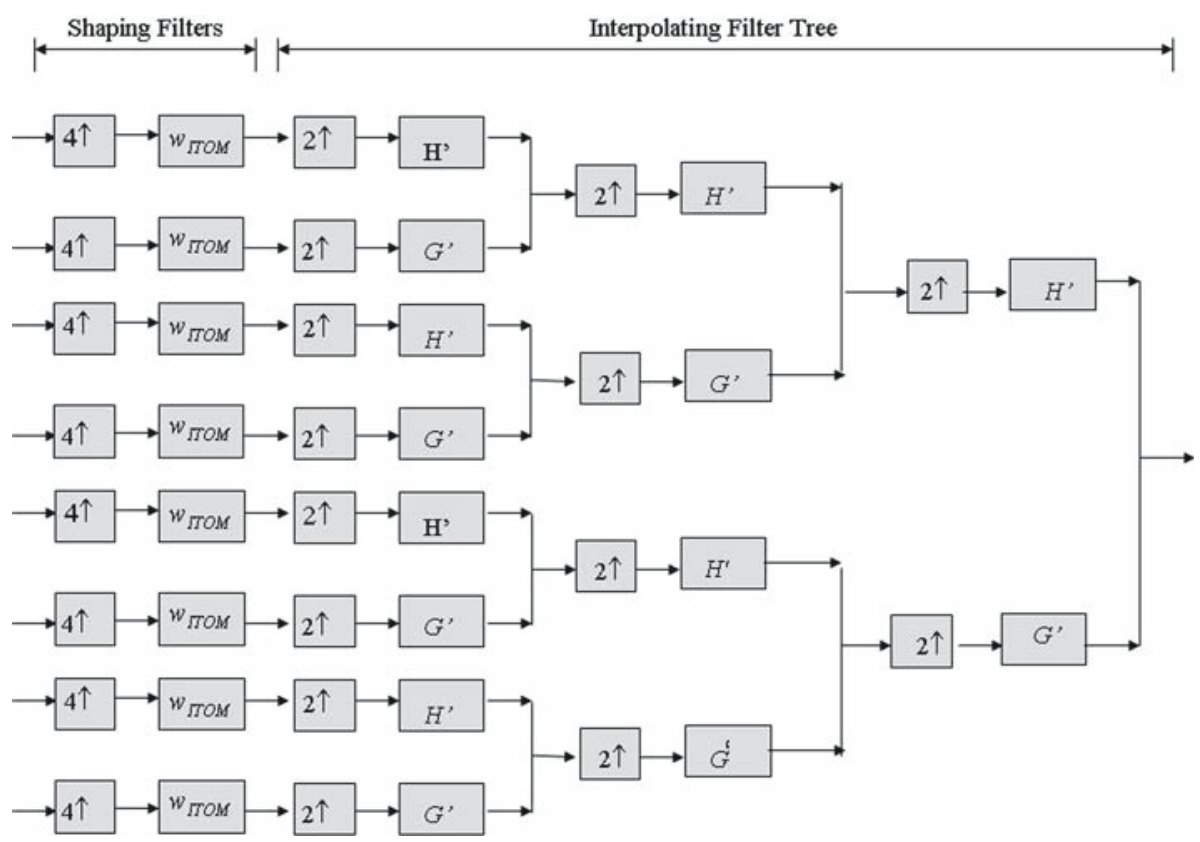

Fig. 18 Modulator of ITOM Interpolated Tree Structure [27]. In the figure $w_{\text {ITOM }}$ is the ITOM shaping filters
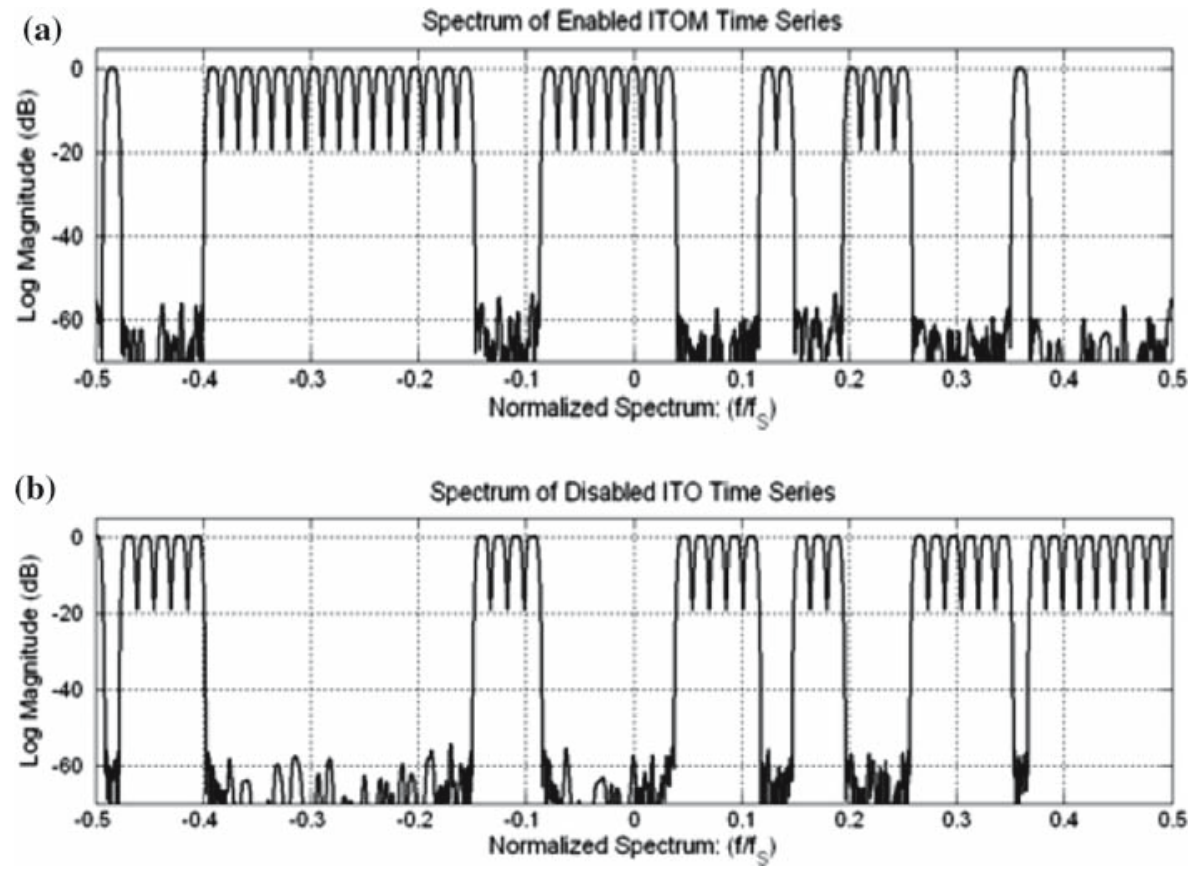

Fig. 19 Illustration of ITOM operation: (a) Spectra of Enabled Spectral Bands of 64-point ITOM; (b) Spectra of Disabled Spectral Bands of 64-point ITOM [27] 
(a)
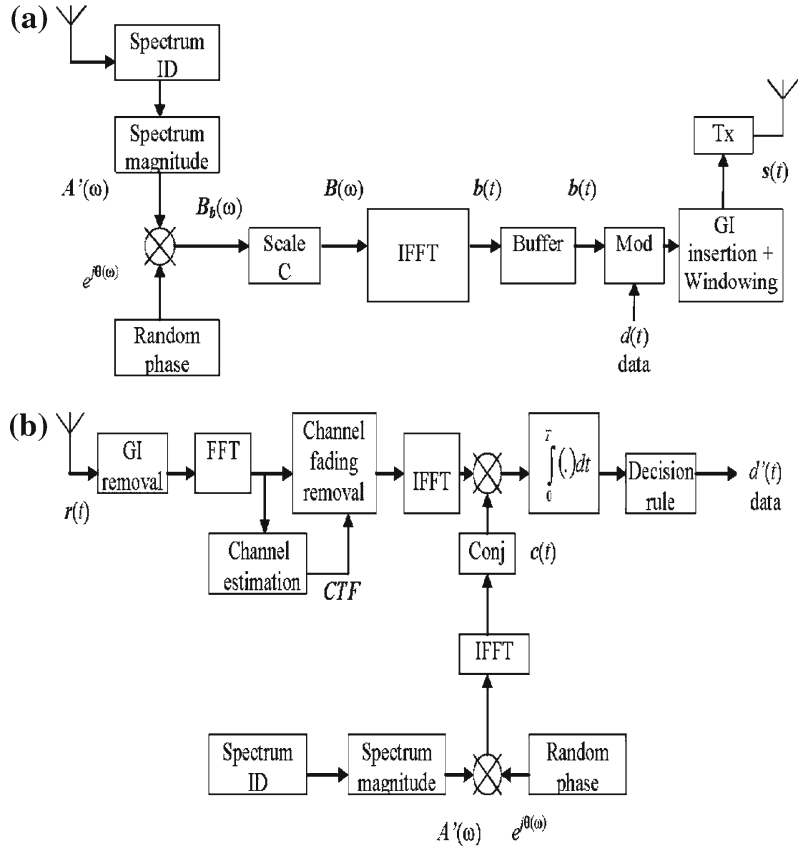

Fig. 20 TDCS (a) Transmitter and (b) Receiver

bandwidth does not contain the LUs band. In [10] the Fourier transform as a tool in shaping the spectrum in TDCS is replaced by the wavelet basis function. The technique is called Wavelet Domain Communication System. The two techniques will be further explored in the following subsections.

\subsection{Transform Domain Communication System (TDCS)}

The TDCS transmitter system is described in Fig. 20a where single carrier is used. A clean spectrum information is derived from $\mathrm{A}^{\prime}=\left\{A^{\prime}\left(\omega_{1}\right), A^{\prime}\left(\omega_{2}\right), \ldots, A^{\prime}\left(\omega_{N F F T}\right)\right\}$, where amplitudes of interfering frequency components exceeding a threshold are set to zero, while the others are set to one. Multi-valued complex pseudorandom (PR) phase vector $\left(e^{j \theta(\omega)}\right)$ is multiplied element by element with $A^{\prime}(\omega)$ to produce the spectral vector $\mathbf{B}(\omega)$. After IFFT operation, a time domain basis function $b(t)$ is derived, which subsequently is stored and modulated by using pulse amplitude modulation (PAM), pulse position modulation (PPM) or cyclic code shift keying (CCSK) where the bits are represented by the cyclic shifted pulse in time. The impact of this zeros insertion can be described from the PSD equation of TDCS,

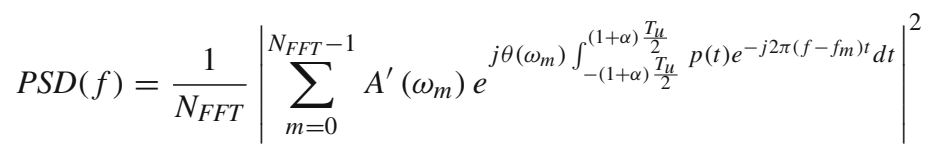

where $T_{u}$ is the useful signal duration, $p(t)$ is the window function, $\alpha$ is the roll off factor of the window, and $\theta\left(\omega_{m}\right)$ is the phase on subband $m$ produced by the random phase module. 
If $p(t)$ is a rectangular window and $\alpha$ is zero, the area within the integral can be replaced by $T_{u} \operatorname{sinc}\left(\left(\pi f-\pi f_{m}\right) T_{u}\right)$. Zero amplitude at the carrier $\omega_{m}$ will make the PSD on that carrier position becoming zero, and due to the orthogonality among carriers the power contributions from the other carriers are also zero. The equation is almost similar to (6), the differences are the power $p_{m}$ and symbol amplitude $A_{m}$ in (6) are replaced by the spectrum information $A^{\prime}\left(\omega_{m}\right)$ and the random phase $\theta\left(\omega_{m}\right)$.

TDCS gives more degree of freedom in choosing window $p(t)$ to lower the sidelobes of its spectrum. Unlike OFDM, the spectrum sidelobes of the window of TDCS do not have to be zero on the frequency spacing interval (in OFDM this is described as the orthogonality between carriers), as long as its sidelobes are very small. Since data detection at the receiver is performed by correlating the received signal with reference signal, as described in Fig. 20b, the designed non-orthogonal spectrum of the transmitted signal will not affect the data detection.

\subsection{Wavelet Domain Communication System (WDCS)}

An offshoot of TDCS is the wavelet domain communication system (WDCS) [10] where the Fourier transform operation in TDCS is replaced by wavelet transformation. Wavelets are used in this scheme to identify and establish an interference-free spectrum. The advantages of using wavelet are [10]:

1. Increased adaptation over a larger class of interfering signals,

2. Finer high-frequency resolution,

3. Allow implementation of $C S$-ary orthogonal signaling

The WDCS uses a packet-based transform to estimate the electromagnetic spectrum [10]. Through the use of adaptive thresholds and notches, sub-bands containing the interference are effectively canceled. From this estimate, a unique communication basis function $A^{\prime}(\omega)$ in the transform domain is generated so that no (or very little) energy-bearing information is contained in the areas occupied by primary users. These functions are then multiplied with a PR phase vector $e^{j \theta(\omega)}$ to generate $B_{b}(\omega)$. The PR code is used to randomize the phase of the spectral components. The resulting complex spectrum is then scaled $C$ to provide the desired energy in the signal spectrum. A time domain version $b(t)$ of the basis function is then obtained by performing an inverse wavelet transfom. The wavelet basis function can be Coifflets, Daubechies, Haar, Symlets or Remez filter as also be applied in WP-MCM. Finally, the basis function is modulated with data using PAM, or PPM, or CCSK modulation and then transmitted. The block diagram of the WDCS transmission process is shown in Fig. 21.

At the receiver the detection is preceded by correlating the received signal with the reference signal in time domain. The receiver structure of WDCS is almost similar to the TDCS, the differences are the FFT and IFFT modules in TDCS that are replaced by discrete wavelet transform (DWT) and inverse discrete wavelet transform (IDWT), respectively.

\section{MIMO for Cognitive Radio}

Due to deactivation of carriers and long windowing duration upon the LUs occupancy, the $\mathrm{CR}$ bit rate is decreased. Multiple transmit antennas can be applied to CR for the purpose of transmitting different data on different antennas to increase the bit rate. The resource loss due to carriers deactivation on LUs band and bit rate loss due to long symbol duration are compensated. The transmitted power is distributed uniformly among the transmit antennas, 


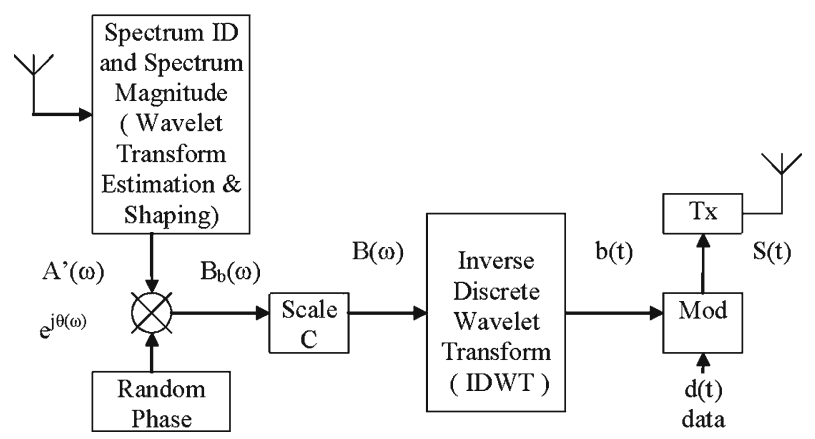

Fig. 21 WDCS Transmitter blocks [10]

so the total power is kept constant. In [48] CR with MC-OFDM and SC-TDCS on MIMO system using V-BLAST Receiver architecture has been evaluated. The results show the bit rate increases with the trade_off of more required pilots to estimate the channel transfer function of each transmit (Tx) and receive (Rx) antenna link. The composition of larger number of receive antenna compared to transmit antenna boosts the BER performance of the CR system. Especially for the TDCS the performance gain is even more significant than MC-OFDM for high SNR. Interesting result of [48] shows that more SNR gain can be achieved by having more Rx antennas when compared to Tx antennas. Furthermore, this SNR gain is more significant when compared to the adaptive bit loading applied to OFDM with spectrum pooling [38].

\section{Beamforming}

Beamforming is formerly a multi access technique in mobile communications that takes the advantage of the position and angle of the other communications partners (nodes). This technique can increase the range between communications nodes, since the signal beam is concentrated (focused) on the intended communication partner hence no energy is wasted to the other direction. In CR system two scenarios can be considered in the application of beamforming: first is the interference avoidance to the LUs by not directing the signal beams to the LUs while occupying the LUs frequency, and second is the communications to multiple CR nodes without interference to each other (no multiple access interference).

In the case where no beamforming is applied, the RU can only occupy the LUs band if its distance to the licensed user is far so that it will not interfere with the LUs signal decoding process. Figure 22a shows how the scenario should be when LU base station (BS) transmits data to LU mobile station (MS) which is located on the border of decodability region (dashed line). The RU can only transmit outside the "no talk zone" (solid line). The shaded region is called "protected region" where the signal decodability of the LUs is guaranteed [49]. Figure 22b depicts the case when beamforming is applied by the RUs, the beams are directed to the intended RU partner, where communication to several RU nodes does not interfere to each other, and beams to the direction of LUs are nullified.

The important process in beamforming is the direction of arrival (DOA) estimation of the incoming signals. DOA estimation with Multiple Signal Classification (MUSIC) is introduced in [50], while Estimation of Signal Parameters by Rotational Invariance Techniques (ESPRIT) is proposed in [51]. These DOA techniques give the angle directions from where 

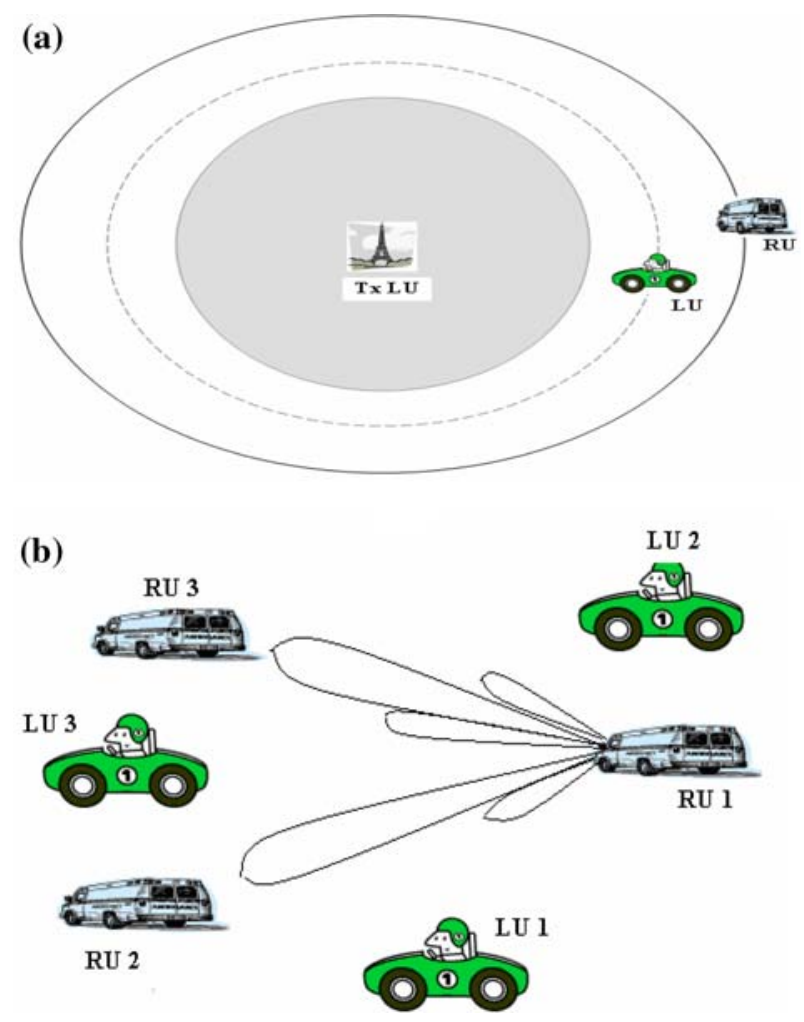

Fig. 22 (a) CR without beam forming [49] (b) CR with beamforming

the LUs signals are coming. An antenna array as well as a baseband model to estimate the direction of the intended partner is shown in Fig. 23a and b, respectively. If the plane wave incident on the array is from an angle $(\theta, \phi)$ relative to the axis of the array, where $\theta$ is the angle of the wave incident with respect to the $z$ axes, while $\phi$ is the angle between the $x$ axis and the map of the wave incident on the $x-y$ plane. If the array elements have uniform gain in all directions, and the spacing between array elements $(\Delta x)$ is small, there will be no amplitude variations between signals received at different elements. All incident fields can be decomposed into a discrete number of plane waves. Other assumptions are well defined in [53]. The received signal will update the weights of the linear array elements and accordingly the maximum main beam of the array will be directed to the desired direction. By pointing the beam to specific angle where the LU signal comes and taking the Fourier transform of the received signal on that direction then the CR node with beamforming capability will have the information regarding the spectrum occupied on specific spatial direction. Accordingly this information is used for transmission of the CR system signal without interfering the LUs within spatial directions and certain frequency band. Recent investigation in [52] shows that the utilization of beamforming by the LUs can enhance the coverage area of the CR system, and the propagation condition between the LUs and CR system affects the CR system's coverage area size. According to [52] for reusable CR coverage area $>70 \%$, a signal to Interference Ratio (SIR) gain of about $14 \mathrm{~dB}$ is achieved by the CR system where the LUs beamwidth is $10^{\circ}$ in vertical plane compared to omni directional beam. 
(a)
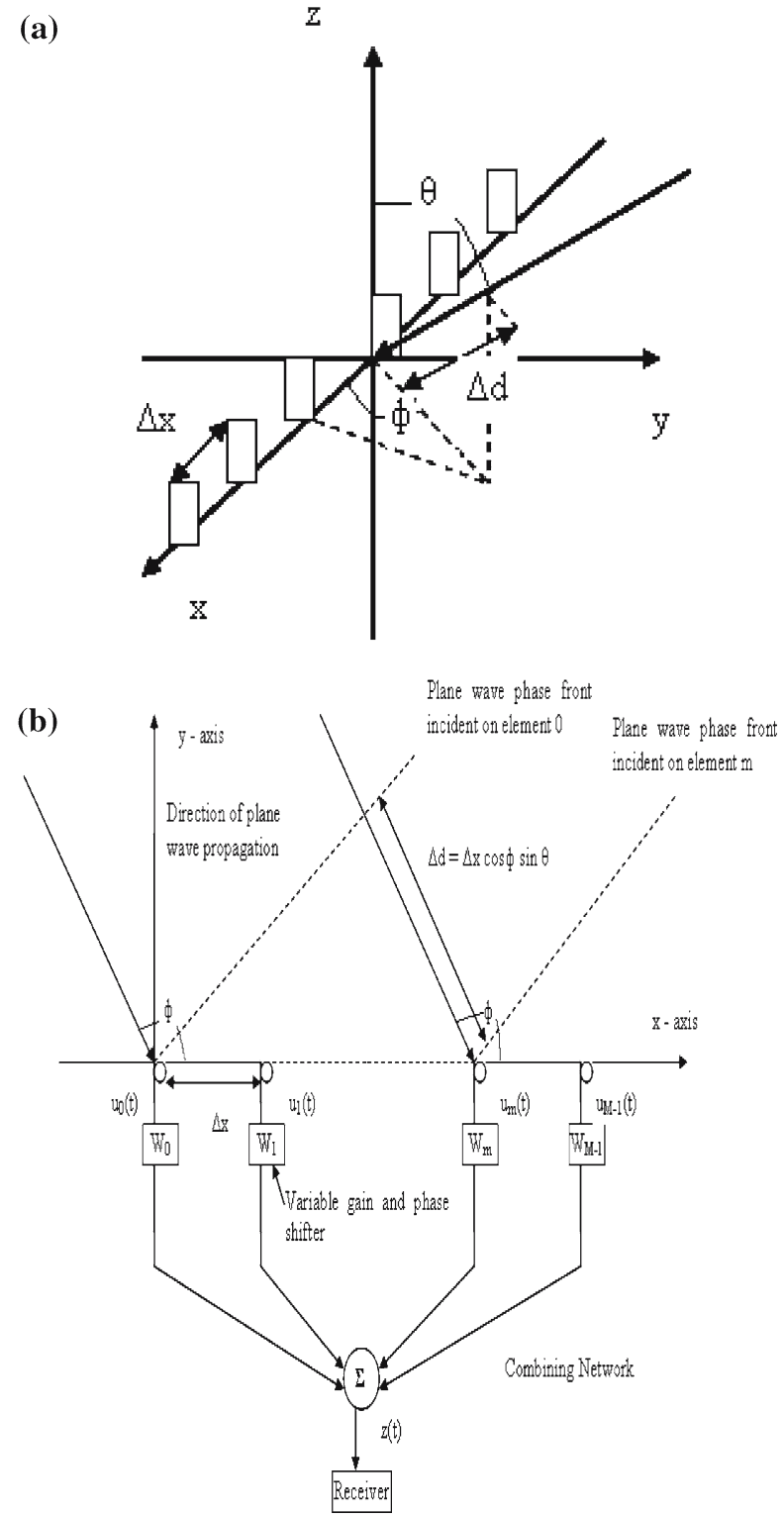

Fig. 23 (a) A linear equally spaced array antenna (b) Baseband model of a linear equally spaced array for estimating Direction of Arrival of a plane wave incident on the antenna elements [53]

\section{Summary}

In this tutorial paper techniques for CR were discussed. Spectrum sensing as the crucial element of cognition in terms of spectrum awareness was reviewed. Adaptive OFDM with Fourier and wavelet basis functions and the combination with spectrum pooling as well as the single carrier modulation with wave shaping technique using Fourier and wavelet 
transform were explained. MIMO technique as a QoS enhancement scheme for CR system was explained and beamforming as a spatial awareness technique in CR was studied.

Spectrum pooling enables the access to spectrum without giving significant interference to the actual license owners. The high sidelobes of OFDM in spectrum pooling can be counteracted by deactivating carriers adjacent to the LUs (non contiguous OFDM), and applying a proper window to the signal in time domain. The loss due to carrier deactivation and long windowed OFDM symbol duration can be compensated by adaptive bit loading so the system still can achieve the target bit rate. Spectrum pooling provides a high degree of freedom in choosing the window to suppress the sidelobes of the signal since the signal orthogonality can be preserved at the receiver by applying the rectangular window implemented by DFT. If target bit rate is the main concern in adaptive bit loading, then groupwise bit loading is the choice, while if target error rate is the constraint, then carrierwise bit loading will be the solution.

Overlapping OFDM symbol transmission is another method in counteracting the loss of bit rate due to the long OFDM window duration. However the starting point of the consecutive OFDM symbols should be taken care of in order to avoid the ISI. Frequency hopping is the solution in very harsh channel environments where propagation condition and LUs access is very severe. Frequency hopping is only conducted when it is necessary.

Wavelet transform is considered to be a better modulation method compared to the Fourier transform method due to its flexibility in shaping the spectrum and its better ICI and ISI suppression. With the proper basis functions in the wavelet transform, the spectrum can be used efficiently; hence bit rate can be maintained or even increased. Furthermore, no guard interval is needed for the wavelet which is another boost for the throughput. A practical utilization of wavelet in CR system is by representing the RU signal as wavelet packet coefficients and by selectively removing those coefficients whose spectra footprint lies in the LU band. In all these techniques, the reliable information from the spectrum sensing unit is essential.

TDCS and WDCS are single carrier transmission techniques in utilizing the spectrum efficiently. The technique provides a degree of freedom in reducing its sidelobes which also directly reduces the interference to LUs band. In TDCS unlike in OFDM, the transmitted signal inter frequency orthogonality is not necessary since the data detection at the receiver is conducted by correlating the received signal with the reference signal. The drawback of the single carrier approach is the achievable data rate which is lower compared to OFDM.

MIMO system boosts the bit rate of CR system with additional computational costs of estimating all of the Tx and Rx channel links. For the improvement of BER of CR system, the usage of more receive antennas (than transmit antennas) is suggested.

Beamforming gives spectrum sharing capability to a wireless system. It also enhances the multiple access capability of the CR system. As beamforming technique can be used by the scanning module to detect the spectrum occupancy in a specific direction, it will also enhance the coverage area of a CR system.

Acknowledgements The authors acknowledge the support of Dutch Adaptive Adhoc Freeband (AAF) project in this research.

Open Access This article is distributed under the terms of the Creative Commons Attribution Noncommercial License which permits any noncommercial use, distribution, and reproduction in any medium, provided the original author(s) and source are credited. 


\section{References}

1. Brodersen, R. W., et al. (2004). CORVUS: A Cognitive Radio approach for usage of virtual unlicensed spectrum. White Paper, Department of Electrical Engineering and Computer Science University of Berkeley, July, 2004.

2. Haykin, S. (2005). Cognitive Radio: Brain empowered wireless communications. IEEE Journal on Selected Areas in Communications, 23(2), 201-220.

3. Moseley, N. A. (2004). Radio resource discovery for ad-hoc wireless networking. M.Sc Thesis, University of Twente, Enschede, Netherland, August 2004.

4. Weiss, T., et al. (2004). Mutual interference in OFDM based spectrum pooling systems. In Proceedings of IEEE Vehicular Technology Conference, VTC Spring 2004, Milan, Italy, May 2004.

5. Cabric, D., Mishra, S. M., \& Brodersen, R. W. (2004). Implementation issues in spectrum sensing for Cognitive Radios. In Proceedings of the 38th ASILOMAR Conference on Signals, Systems, and Computers (Vol. 1, pp. 772-726), Pacific Grove, CA, USA, November 2004.

6. Hoeksema, F. W., Heskamp, M., Schiphorst, R., \& Slump, C. H. (2005). A node architecture for disaster relief networking. In Proceedings of the 1st IEEE International Symposium on new Frontiers in Dynamic Spectrum Access Networks (DySPAN'05), pp. 577-584, Baltimore, USA, November 2005.

7. Jamin, A., \& Maehoenen, P. (2004). Wavelet packet modulation for wire-less communications. Wireless Communications and Mobile Computing Magazine, Wiley Interscience, 5, 123-137.

8. Negash, B. G., \& Nikookar, H. (2000). Wavelet based multicarrier transmission over multipath wireless channel. IEE Electron. Letters, 36(21), 1787-1788.

9. Chakravarthy, V., et al. (2005). Cognitive Radio-An adaptive waveform with spectrum sharing capability. In IEEE Wireless Communications and Networking Conference, New Orleans, USA, March 2005.

10. Lee, M. J. (2002). Wavelet domain communication system (WDCS): Packet-based wavelet spectral estimation and M-ary signaling. Master thesis, Air Force Institute of Technology, Ohio, USA, March 2002.

11. Cabric, D., Tkachenko,A., Brodersen, R. W. (2006). Spectrum sensing measurements of Pilot, Energy, and collaborative detection. In Proceedings of IEEE Military Communications Conference (MILCOM), Washingthon, D.C., USA, October 2006.

12. Tian, Z., \& Giannakis, G. B. (2006). A wavelet approach to wideband spectrum sensing for Cognitive Radios. In Proceedings of International Conference on Cognitive Radio Oriented Wireless Networks and Communications, Greece, June 8-10, 2006.

13. Hur, Y., Park, J., Woo, W., Lim, K., Lee, C.-H., Kim, H. S., \& Laskar, J. (2006). A wideband analog multi-resolution spectrum sensing (MRSS) technique for cognitive radio (CR) systems. In Proceedings of IEEE International Symposium Circuit and System, pp. 4090-4093, May 21-24, 2006.

14. Cabric, D., \& Brodersen, R. W. (2005). Physical layer design issues unique to Cognitive Radio systems. In Proceedings of IEEE Personal Indoor and Mobile Radio Communications (PIMRC), September 2005.

15. Hunziker, T., \& Dahlhaus, D. (2001). Adaptive OFDM transmission for broadband fixed wireless access systems. In Proceedings of 6th International OFDM Workshop (InOWo), pages 5-1-5-4, Hamburg, Germany, 2001.

16. Hunziker,T., \& Dahlhaus, D. (2003). Optimal power adaptation for OFDM systems with ideal bit-interleaving and hard decision decoding. In Proceedings of IEEE International Conference Communications (ICC), 3392-3397, May 2003.

17. Park,C. S., \& Lee,K. B. (2004). Transmit power allocation for BER performance improvement in multicarrier systems. In IEEE Transactions on Communications, 52(10), 1658-1663.

18. Dagres, I., \& Polydoros, A. (2005). Mode adaptation combined with power allocation for guaranteed QoS constraints in COFDM Transceivers. In Proceedings of WPMC'05, Aalborg, Denmark, pp. 726-729, September 2005.

19. Kaemarungsi, K., \& Krishnamurthy, P. (2003). On the use of adaptive OFDM to preserve energy in ad hoc wireless networks. In Proceedings of 13th MPRG/Virginia Tech Symposium on Wireless Personal Communications, USA, June 2003.

20. Krongold, B. S., et al. (2000). Computationally efficient optimal power allocation algorithms for multicarrier communications systems. In IEEE Transactions on Communications, 48(1), 23-27.

21. Nikookar, H., \& Prasad, R. (1997). Wave shaping of multicarrier signal for data transmission Over wireless channels. In IEEE 6th International Conference on Universap Personal Communications Record (ICUPC), pp 173-177.

22. Tan,P., \& Beaulieu, N. C. (2004). 'Reduced ICI in OFDM systems using the better than raised-cosine pulse. IEEE Communications Letter, 8(3), 135-137.

23. Assalini,A., \& Tonello, A. M. (2004). Improved nyquist pulses. In IEEE Communicaions Letters, 8(2), 87-89. 
24. Muschalik, C. (1996). Improving An OFDM reception using an adaptive nyquist windowing. In IEEE Transactions on Consumer Electronics, 42(3), 259-269.

25. Witrisal, K. (2002). OFDM air interface design for multimedia communications. Ph.D. Thesis, Delft University of Technology, April 2002.

26. Nikookar, H., \& Prasad, R. (1997). Optimal waveform design for multi-carrier transmission over a multipath channel. In Proceedings of 47th IEEE Vehicular Technology Conference, Phoenix, Arizona, USA, May 4-7, 1997.

27. Harris, F., \& Kjeldsen, E. (2006). A novel interpolated tree orthogonal multiplexing (ITOM) scheme with compact time-frequency localization: An introduction and comparison to wavelet filter banks and polyphase filter banks. In Software Defined RadioTechnical Conference and Product Exposition, Orlando, Florida, USA, November 2006.

28. Hanzo, L., et al. (2002). Adaptive wireless transceivers. Chichester, England: Wiley

29. Reddy, S. B., et al. (2003). An efficient blind modulation detection algorithm for adaptive OFDM systems. In Proceedings IEEE Vehicular Technology Conference (VTC) (Vol. 3, pp. 1895-1899). Orlando, USA, October 2003.

30. Budiarjo, I., Heskamp, M., Zhang, Q., Nikookar, H., \& Kokkeler, A. J. (2007). D.3.41 Radio system: Second phase verification and advances in architecture and algorithms. Adaptive Ad-hoc Freeband (AAF), July 2007, The Netherlands.

31. Barreto,A. N., \& Furrer, S. (2001). Adaptive bit loading for wireless OFDM systems. Proceedings of the 12th IEEE International Symposium Personal, Indoor, Mobile Radio Communication (Vol. 2, pp. 88-92). San Diego, CA, USA, October 2001.

32. Chow, P. S., et al. (1995). A practical discrete multitone transveiver loading algorithm for data transmission over spectrally shaped channels. IEEE Transactions on Communications 43(2), 773-775.

33. Gruenheid, R., et al. (2001). A blockwise loading algorithm for the adaptive modulation technique in OFDM systems. In Proceedings of VTC, 2, 948-951.

34. Gruenheid, R., et al. (2000). Adaptive modulation for the HIPERLAN/2 air interface. In Proceedings of the 5th International OFDM Workshop, Hamburg, Germany.

35. Lei,M., \& Zhang, P. (2003). Subband and Power Loading for Adaptive OFDM. In Proceedings VTC (Fall) (Vol. 3, pp. 1482-1486). Orlando, USA, October 2003.

36. Budiarjo, I., Nikookar, H., \& Ligthart, L. P. (2005). An overview of adaptive OFDM in the context of Cognitive Radio. In Proceedings of the 12th IEEE Symposium on Communications and Vehicular Technology (SCVT), Enschede, The Netherlands, November 2005.

37. Budiarjo, I., Nikookar, H., \& Ligthart, L. P. (2006). Performance evaluation of adaptive bit loading for OFDM with channel estimation using $2 \times 1-D$ wiener filter. In Proceedings of International Symposium on Wireless Personal and Multimedia Communications, San Diego, CA, USA, September 2006.

38. Budiarjo, I., Nikookar,H., \& Ligthart, L. P. (2006). Combined spectrum pooling and adaptive bit loading for Cognitive Radio OFDM based system. In Proceedings of the 13th IEEE SCVT, Liege, Belgium, November 2006.

39. Mutti, C., et al. (2004). Adaptive coding based on LDPC Codes for OFDM Systems with HD decoding. In Proceedings of the 13th IST Mobile \& wireless Communications Summit, Lyon, France, June 2004.

40. Jetlund, O., et al. (2002). Rate-adaptive coding and modulation with LDPC component codes. In COST 273 TD(02)-108, 5th Management Committee Meeting COST Action 273, Towards Mobile Broadband Multimedia Networks, Lisbon, Portugal, September 2002.

41. Vucetic, B. (1991). An adaptive coding scheme for time varying channels. In IEEE Transactions on Communications, 39, 653-663.

42. Keller, T., et al. (2000). Adaptive rate RRNS coded OFDM transmission for mobile communication channels. In Proceedings of IEEE 5 VTC, Tokyo, Japan, May 2000.

43. Budiarjo, I., Nikookar, H., \& Ligthart, L. P. (2007). Performance evaluation of OFDM based Cognitive Radio system with wiener filter channel estimation using frequency hopping GSM channel model at $900 \mathrm{MHz}$. In Proceedings of the 10th European Conference on Wireless Technology (ECWT) (pp. 74-77). Munich, Germany, October 2007.

44. Bouwel, C. V., Potemans, J., Schepers, S., Nauwelaers, B., \& Capelle, A. V. (2000). Wavelet packet based multicarrier modulation. Proceedings IEEE Benelux Symposium on Communications and Vehicular Technology, Leuven, Belgium, October 2000.

45. Jensen, A., \& Cour-Harbo, A. L. (2001). Ripples in mathematics: The discrete wavelet transform, Springer, 2001.

46. Rioul, O., \& Duhamel, P. (1994). A Remez exchange algorithm for orthonormal wavelets. IEEE Transactions on Circuits Systems-II, 41(8), 550-560.

47. Lakshmanan, M. K., Budiarjo, I., \& Nikookar, H. (2007). Maximally frequency selective wavelet packets based multi-carrier modulation scheme for Cognitive Radio systems. In Proceedings of the 50th IEEE 
Global Communications Conference (Globecom) (pp. 4185-4189). Washingthon DC, USA, November 2007.

48. Budiarjo, I., Nikookar, H., \& Ligthart, L. P. (2007). Cognitive Radio with single carrier TDCS and multicarrier OFDM Approach with V-BLAST receiver in Rayleigh fading channel. In Proceedings of the 2nd International Conference on Cognitive Radio Oriented Wireless Networks and Communications (CrownCom), Orlando, USA, August 2007.

49. Sahai, A., Hoven, N., \& Tandra, R. (2004). Some Fundamental Limits on Cognitive Radio. In 42nd Allerton Conference on Communication, Control, and Computing, Monticello, IL, October 2004.

50. Schmidt, R. O. (1986). Multiple Emitter Location and Signal Parameter Estimation. In IEEE Transactions on Antennas and Propagation, AP-34(3).

51. Paulraj, A., Roy, R., \& Kailath, T. (1986). A subspace rotation approach to signal parameter estimation. Proceedings of IEEE, 74(7), 1044-1045.

52. Nishimori, K., et al. (2006). Spatial availability for Cognitive Radio system under directional interference. In Proceedings of International Symposium on Wireless Personal and Multimedia Communications, San Diego, CA, USA, September 2006.

53. Liberti, J. C., Jr., \& Rappaport, T. S. (1999). Smart antennas for wireless communications, Prentice Hall, NJ, USA.

\section{Author Biographies}

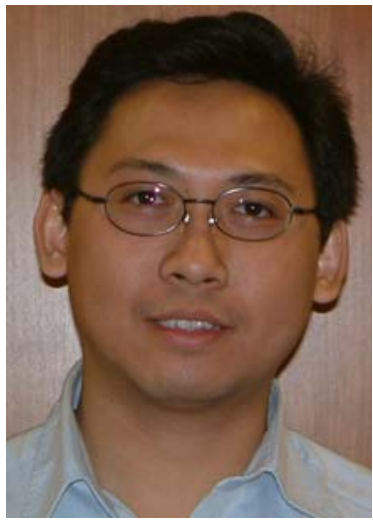

I. Budiarjo received his Bachelor of Engineering (Sarjana Teknik) degree in Electronics from Bandung Institute of Technology, Indonesia, in 2001. He got his Master of Science degree in Communications Engineering from Munich University of Technology (TU Muenchen), Germany, in 2003. He is now working toward a PhD in International Research Centre for Telecommunications-Transmission and Radar, Delft University of Technology (TU Delft), The Netherlands. His research interests focus on adaptive signal processing for Cognitive Radio, including CDMA, OFDM, wavelet packet modulation, MIMO systems, beamforming and embedded system for cognitive radio applications.

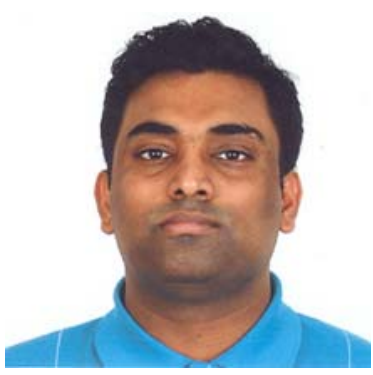

M. K. Lakshmanan was born in Chennai, India, in 1979. He received the $\mathrm{BE}$ (with distinction) in electrical engineering from the University of Madras, Chennai, India, in 2000. In 2000, he joined the Indian Software firm, Polaris Software Labs Ltd., where he wrote software for Telecommunication applications. At Polaris, he was awarded the "On The Spot Of Excellence Award" for his efforts. In 2003, he moved to the Indian Institute of Technology-Madras, India, to lead a team of junior researchers to develop and establish a wireless communications network for rural connectivity. In 2004, he was granted the Royal Dutch/Shell Chevning scholarship to pursue a Masters program in Telecommunications at the Delft University of Technology (TU Delft). He completed his Masters (with CUM LAUDE) in the 2006 and continued with his doctoral studies at TU Delft where he is currently conducting research in the fields of wavelets, signal processing and Wireless Communications at the International Research Center for Telecommunications Transmission and Radar (IRCTR). In December 2007 he won the BEST STUDENT PAPER award at The 10th International Symposium on Wireless Personal Multimedia Communications, Jaipur, India. 


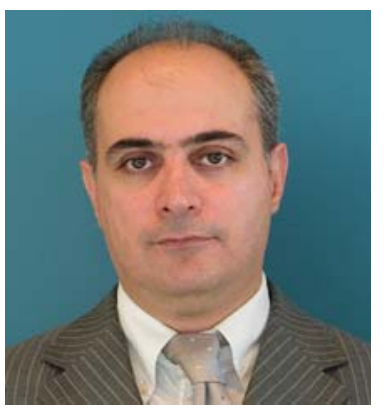

H. Nikookar received his $\mathrm{PhD}$ in Electrical Engineering from Delft University of Technology (TUDelft), The Netherlands, in 1995. He is an Associate Professor at the International research Centre for Telecommunications and Radar (IRCTR) of the Department of Electrical Engineering, Mathematics and Computer Science of TUDelft. He is also the coordinator of the Radio Advanced Technologies and Systems (RATS) program of IRCTR. Dr. Nikookar has a rich experience in conducting research in many aspects of wireless communications, including wireless channel modelling, UWB, MIMO, multicarrier transmission, Waveletbased OFDM and Cognitive Radio. He is a senior member of the IEEE. 